\title{
Was von Entscheidungsträgern erwartet wird: Das Modell der Strategischen Führungskompetenzen
}
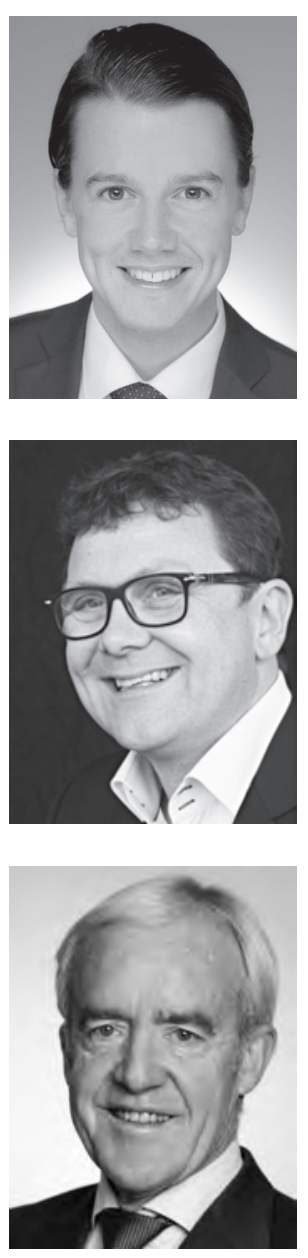

\section{Sebastian Bekemeier, Timo Meynhardt und Günter Müller-Stewens}

Der Bedarf an Führungskräften, die eine Kompetenz zur strategischen Führung besitzen, ist heute größer denn je. Sie sind es, die den Wandel, der derzeit nahezu allerorts so fundamental stattfindet, gestalten und umsetzen müssen. Von ihnen wird erwartet, dass sie strategische Initiativen unternehmerisch managen, mit denen die technologischen, demografischen, geopolitischen und sozialen Veränderungen in ihren Strategien antizipativ aufgegriffen werden und die das Unternehmen zukunftsfähig machen. Sie müssen sich dazu in den heutigen mehrdimensionalen Organisationsstrukturen mit ihren unzähligen Schnittstellen zurechtfinden und durchsetzen können. Auch müssen sie in der Lage sein, Netzwerke auf Augenhöhe mit den strategischen Partnern unternehmerisch nutzbar zu machen. Das heißt, es reicht vielerorts nicht mehr aus, nur ein Manager zu sein, der seine definierte Aufgabe verwaltet; für den Wandel braucht es Strategic Leaders. In diesem Beitrag wird ein eigenes «Modell der Strategischen Führungskompetenzen» entwickelt und es werden Wege zu seiner Anwendung aufgezeigt.

The need for leaders with leadership skills is now greater than ever. They must shape and implement the change that is so fundamental almost everywhere today. They are expected to entrepreneurially manage strategic initiatives that anticipate technological, demographic, geopolitical and social changes in their strategies and make the company fit for the future. They have to be able to navigate and assert themselves in today's multi-dimensional organizational structures with their innumerable interfaces. In addition, they must be able to make networks on an equal footing with the strategic partners entrepreneurial. This means that in many places it is no longer enough to be just a manager who manages his defined task; change requires strategic leaders. This paper develops a "Model of Strategic Leadership Competences" and shows ways to apply it.

Strategisches Management, strategische Führung, Entscheidungsträger, Kompetenzen, Kompetenzmodelle, Assessments, Executive Search

Strategic management, strategic leadership, decision maker, competences, competence models, assessments, executive search 
Es ist leicht gesagt, dass Führungskräfte die Kompetenz zur strategischen Führung haben müssen. Doch wie definiert sich diese Kompetenz genau? Welche Teilkompetenzen müssen besondere Beachtung finden, damit Führungskräfte der Erwartungshaltung an eine strategische Denk- und Arbeitsweise gerecht werden? Nach welchen Kriterien soll verfahren werden, wenn zum Beispiel eine Führungskraft für eine strategisch bedeutsame Position bewertet und ausgewählt wird?

Um diese Fragen beantworten zu können, wurde in einem Forschungsprojekt das Ziel verfolgt, das Konstrukt „strategische Führungskompetenz“ so zu bestimmen, dass Organisationen in die Lage versetzt werden, Bewerber und Mitarbeiter - bis hin zu ganzen Managementteams oder -ebenen - hinsichtlich ihrer strategischen Führungskompetenz zu auditieren und zu entwickeln.

Dazu soll zuerst ein Blick auf die Kompetenzforschung geworfen werden. Dabei handelt es sich um ein äußerst umfassendes Forschungsfeld mit einer langen Tradition, das in der hier gebotenen Kürze unmöglich auch nur annähernd vollständig besprochen werden kann. Doch reichhaltige Fußnoten sollen interessierten Lesern bei der Vertiefung weiterhelfen. Im zweiten Abschnitt wenden wir uns dann der konkreten Entwicklung eines eigenen Modells der Strategischen Führungskompetenzen (MSF) zu, um im dritten Abschnitt in seine Anwendung einzuführen. Ein solches Modell hilft uns bei der Personalarbeit mit strategischen Führungskräften, es kann uns aber auch bei der eigenen Entwicklung als Führungskraft Orientierung geben. Denn indem ein solches Modell in einer Organisation transparent gemacht wird, macht man es auch für den Einzelnen und dessen Entwicklung zugänglich. Im Gegensatz zu Charakterzügen oder Eigenschaften, die nur sehr bedingt gezielt formbar sind, können Kompetenzen über die Zeit gelernt und (weiter-)entwickelt werden.

\section{Auf dem Weg zum Kompetenzmodell}

Wenn gesagt wird, dass für den Erfolg und das Überleben von Unternehmen die frühzeitige Anpassung der Strategien an ein sich veränderndes Umfeld kritisch ist, dann gilt dies logischerweise auch für die Kompetenzen der Führungskräfte, die die strategischen Entscheidungen für derartige Anpassungen treffen (Müller-Stewens 2019) ${ }^{1}$.

Manager haben Wahlmöglichkeiten, das heißt, sie sind nicht hilflos den Veränderungen ihres Umfelds ausgesetzt. In diesem Sinne sind Unternehmen auch Spiegelbilder ihrer strategischen Entscheidungsträger. So prägen ihre Einstellungen, Vorlieben, Erfahrungen oder Kompetenzen auch ihre Interpretationen des Umfelds und damit ihre Entscheidungen. Ihr intellektuelles und soziales Kapital, das sie in ihre Beziehungen einbringen, machen einen wesentlichen Unterschied im Wettbewerb aus (Reed/Lubatkin/Srinivasan 2006).

Betrachten wir die Veränderungen, mit denen sich die Strategiearbeit über die vergangenen Jahrzehnte konfrontiert sah und auch zukünftig sein wird, so ist es naheliegend, dass die zu ihrer Verrichtung erforderlichen Kompetenzen sich analog mitentwickeln müssen. Während es in den 1960er Jahren noch die Planungskompetenz war und in den 1970er Jahren erkannt wurde, dass es auch eine Implementierungskompetenz braucht, hat sich mit der deutlich komplexer gewordenen Strategiearbeit das Spektrum der erforderlichen

1 Vgl. auch Finkelstein/Hambrick/Cannella (2009). Sie gehen deutlich über die in der Topmanagementteam-Forschung lange dominanten demografischen Charakteristika hinaus. 
Bekemeier/Meynhardt/Müller-Stewens | Was von Entscheidungsträgern erwartet wird

Kompetenzen bis heute immer weiter verändert und ausdifferenziert. Auf die Frage, welche dies genau sind, versuchen Kompetenzmodelle eine Antwort zu geben.

In der Unternehmenspraxis treffen wir bereits eine sehr große Anzahl verschiedener Kompetenzmodelle für Führungskräfte an, wie z.B. für Controller. ${ }^{2}$ Doch viele davon sind wenig ausgereift, wissenschaftlich nicht ausreichend fundiert und nur selten stringent implementiert (Sudsakorn/Swierczel 2009). Insbesondere die uns hier interessierende Frage nach den Kompetenzen für eine erfolgreiche strategische Führungsarbeit wird meist nur oberflächlich als Teil eines ganz allgemeinen Führungsmodells betrachtet. Sie wird zwar häufig direkt oder indirekt gestellt, aber selten so präzisiert und ausdifferenziert, dass daraus ein systematisches und wohlfundiertes Vorgehen abgeleitet werden kann (Erpenbeck/von Rosenstiel/Grote 2013).

Diese Aussage trifft auch auf die Forschung zu. Dort ist zwar ein wachsendes Interesse an der Mikroebene der einzelnen Akteure zu beobachten ${ }^{3}$, doch die Einnabme einer reinen Kompetenzperspektive auf die strategische Führungskraft ist bislang nicht erfolgt. Meist wird sie unter den Kompetenzen eines General-Managers knapp subsummiert.

Bevor es in Abschnitt 2 um die Herleitung des angekündigten MSF geht, gilt es, vorab zu klären, was wir genau unter einer Kompetenz und einem Kompetenzmodell verstehen.

\subsection{Zum Verständnis von Kompetenzen}

Die Diskussion zu Kompetenzen ist sehr umfassend und divergent. ${ }^{4}$ Im Folgenden setzen wir uns mit dem generellen Verständnis von Kompetenzen auseinander, um uns dann spezifischer mit der Kompetenz zur strategischen Führung zu befassen.

Unter einer strategische Führungskompetenz verstehen wir die Selbstorganisationsdisposition einer Führungskraft, die ihr ein auf ein bestimmtes Leistungsziel ausgerichtetes, erfolgreiches Verhalten ermöglicht. ${ }^{5}$ Diese Definition soll nun begründet werden.

\section{(1) Allgemeine Merkmale von Kompetenzen}

Unserer Modellentwicklung und -anwendung liegen folgende Merkmale von Kompetenzen zugrunde:

- Disposition: Kompetenzen bei handelnden Personen sind erlernte Dispositionen zur Selbstorganisation. Sie bilden die Voraussetzung dafür, eine entsprechende Leistung hervorzubringen, stellen also selbst keine Leistung dar. Deshalb sind Kompetenzen auch nicht direkt beobachtbar. Auf sie lässt sich nur indirekt über sichtbare (Ergebnisse von) Handlungen zurückschließen. Es findet eine Zuschreibung durch den Beobachter statt.

2 Vgl. zum Beispiel das für die Position von Controllern auf wissenschaftlicher Grundlage entwickelte Kompetenzmodell bei International Group of Controlling (Hrsg., 2015).

3 Vgl. zum Beispiel Johnson/Melin/Whittington (2003), S. 14, zur damit einhergehenden Strategy-asPractice-Perspektive.

4 Vgl. zu einer ausführlichen Auseinandersetzung mit dem Kompetenzbegriff Bekemeier (2016), Abschnitt 2.1. Vgl. zu dieser Diskussion aber auch Boyatzsis (2009), Erpenbeck/von Rosenstiel (2007), Wunderer/Bruch (2000) und Scherm (2014). Insbesondere auf dieser Literatur baut auch der vorliegende Text auf. Wichtige Grundlagenarbeiten stammen insbesondere von Boyatzis (1982), McClelland (1973) und Spencer/Spencer (1993).

5 Diese Definition erfolgt in Anlehnung an Erpenbeck/von Rosenstiel (Hrsg., 2007). Vgl. ähnlich Scherm (2014), S. 21.

Die Unternehmung, 74. Jg., $1 / 2020$ 
- Leistungsbezug: Kompetenzen werden immer in Verbindung zu bestimmten Leistungen gesehen und damit auch aus ihnen abgeleitet. Mit einer solchen relationalen Betrachtung wird indirekt unterstellt, dass das ausgeprägte Vorhandensein einer bestimmten Kompetenz sowie deren Anwendung mit hoher Wahrscheinlichkeit wesentlich zu einer erfolgreichen Bewältigung des entsprechenden Problems beitragen. Allein das Vorhandensein der Kompetenz reicht demnach nicht aus. Es braucht auch die Bereitschaft und das Vermögen zu deren Realisierung, das heißt, sie so einzusetzen, dass es zur angestrebten Leistung kommt.

- Komplexität: Kompetenzen werden insbesondere zur Bewältigung komplexer und offener Problemstellungen seitens der Entscheidungsträger benötigt. Sie sind Selbstorganisationsdispositionen, die im Sinne einer Lernfähigkeit den Umgang mit bisher nicht bekannten Herausforderungen ermöglichen. ${ }^{6}$ Diese Komplexität macht den Unterschied zu Qualifikationen aus, die für klar definierte und einfache Probleme ausreichen. Kompetenzen werden als Voraussetzung dafür gesehen, dass eine Führungskraft auch zukünftig erfolgreich in ihrer Funktion tätig sein kann - und dies nicht nur adaptiv, sondern proaktiv und kreativ.

- Divergenz: Für strategische Problemstellungen gibt es keine vorgefertigten Lösungsmuster. Deshalb sind mehrdimensionale Problembewältigungsstrategien gefragt.

- Wertebezug: Für ein erfolgreiches Entscheiden unter hoher Unsicherheit reichen bestimmte Fertigkeiten und Wissen nicht aus. Angesichts der gegebenen Komplexität sind immer auch Bewertungen gefragt, die auf der Basis der verinnerlichten Werte und Einstellungen der Entscheidungsträger beruhen. Sie nehmen in Situationen hoher Selbstorganisation eine ordnende Rolle ein.

- Vielgestaltig: Eine Kompetenz setzt sich aus verschiedenen Komponenten beziehungsweise psychologischen Merkmalen zusammen. Dazu zählen bestimmte Fähigkeiten, Fertigkeiten, Wissensbestandteile, Persönlichkeitseigenschaften und Motive.

- Entwicklungsfähigkeit: Kompetenzen haben einen dynamischen Charakter. Sie sind auf der Basis von Lernerfahrungen, Trainings oder Ähnlichem erlern- und entwickelbar, werden über Erfahrungen verdichtet und konsolidiert, können aber auch wieder verloren gehen. Daraus ergibt sich auch, dass Kompetenzen in graduell unterschiedlichen $\mathrm{Ni}$ veaus vorliegen.

\section{(2) Die Kompetenz zur strategischen Führung}

Der Begriff der strategischen Führung bezieht sich hier zuerst auf die einzelne strategische Führungskraft, die mehr als nur führt, vielmehr auch über substanzielle Entscheidungsbefugnisse und Verantwortlichkeiten verfügt. In dieser Führungskraft sollten auf der ober(st)en Ebene einer Organisation Strategie und Führung vereint sein.

In diesem Sinne verstehen wir unter strategischer Fübrung die Befähigung einer Führungskraft, ein System der Wertgenerierung und die dazugehörigen Aspekte proaktiv so zu managen, dass sich die Organisation in eine erfolgreiche Zukunft entwickelt. Dafür muss

6 Vgl. zur Unterstellung der relativen Objektbereichsunabhängigkeit (Heyse/Erpenbeck 2009). 
diese Führungskraft in der Lage sein, das Commitment der Mitarbeitenden in der Organisation für die Umsetzung der Strategie zu gewinnen. ${ }^{7}$

Dieser Definition kann entnommen werden, dass es nicht ausreicht, ein herausragender strategischer Denker zu sein. Strategic Leadership ist mehr, als nur die Strategie an das sich verändernde externe Umfeld anzupassen. Es geht auch darum, sich selbst als strategische Führungskraft auf das soziale Umfeld einzulassen. ${ }^{8}$ Damit wird strategische Führung als prinzipiell soziale und politische Aktivität aufgefasst, sodass eine reine Betrachtung des Individuums nicht ausreicht (Finkelstein/Hambrick/Cannella 2009, 5).

Daraus kann geschlossen werden, dass eine strategische Führungskraft in der Lage sein muss, unterschiedliche Rollen in sich zu vereinen, für die es unterschiedlicher Kompetenzen bedarf. Insbesondere ist dies die Vereinigung einer strategisch-visionären Rolle und einer führungs- und umsetzungsorientierten. Je nach Aufgabe können sich Verantwortlichkeiten und Rollen in der Gruppe der Führungskräfte verändern.

Eine strategische Führungskraft muss in der Lage sein, eine Gemeinschaft von Kollegen zu schaffen, die sich im Dialog mit den Anspruchsgruppen befindet, denen gegenüber sie Verantwortung übernommen und ein Nutzenversprechen abgegeben hat. Diese Gemeinschaft gilt es eher zu stimulieren, weniger zu kontrollieren. Dann basiert eine strategische Führung auf miteinander geteilten Einsichten, Wissen und Verantwortlichkeiten für zu erreichende Ergebnisse.

Aufgrund eines solch breiten Rollenverständnisses ist es nicht verwunderlich, dass die in der Literatur anzutreffenden Listen der Kompetenzen, die zur erfolgreichen Ausübung dieser Rollen erforderlich sind, sehr divers ausfallen und keine breite empirische Basis haben. ${ }^{9}$

Die Strategieforschung setzte sich ebenfalls mit dem Thema Kompetenzen auseinander, allerdings bezogen auf das Unternehmen und nicht auf die einzelnen Akteure. Dies begann mit dem ressourcenbasierten Ansatz ${ }^{10}$, der Unterschiede in der Performance mit einer heterogenen Verteilung von Ressourcen und Fähigkeiten zwischen Wettbewerbern erklärt. Ressourcen sind die Quelle für Wettbewerbsvorteile, wenn sie selten, wertvoll und schwer imitier- oder substituierbar sind. Diese Überlegungen werden später im Kernkompetenzen-Ansatz aufgegriffen (Prahalad/Hamel 1990). Hier geht es um die Fähigkeit des Unternehmens, verschiedene Ressourcen, Fähigkeiten und Technologien unternehmensweit zu etwas Ganzem und Neuem zu kombinieren und zu bündeln, woraus eine wesentliche Quelle für den Unternehmenserfolg erwachsen soll. Die bislang offene Frage, wie es zu solchen herausragenden Kompetenzen eines Unternehmens kommt, wird im Ansatz der Dynamic Capabilities aufgegriffen (Teece/Pisano/Shuen 1997). Danach braucht es die Fähigkeit, die eigenen Kompetenzen - der dynamischen Umwelt entsprechend - durch eine Rekonfigurati-

7 Ireland/Hitt (2005), S. 63, definieren zum Beispiel wie folgt: „Strategic Leadership is defined as a person's ability to anticipate, envision, maintain flexibility, think strategically, and work with others to initiate changes that will create a viable future for the organization."

8 Vgl. ähnlich Whittington (2001), S. 55.

9 Bei Ireland/Hitt (2005) werden zum Beispiel folgende sechs Teilkompetenzen aufgezählt: (1) Herausarbeiten und Miteinander-Teilen einer gemeinsam getragenen Vision und Mission; (2) Ausschöpfen und Erhalten von Kernkompetenzen; (3) Entwicklung von Human Capital; (4) Erhalt einer effektiven Organisationskultur; (5) Betonung ethischer Praktiken und (6) Etablierung ausbalancierter organisatorischer Steuerung, das heisst strategisch und finanziell zu steuern ohne Eigenmotivation, Flexibilität und Kreativität einzuschränken.

10 Der ressourcenbasierte Ansatz setzte auf den Überlegungen von Penrose (1959) auf.

Die Unternehmung, 74. Jg., $1 / 2020$ 
on der entsprechenden Fähigkeiten, Fertigkeiten und Ressourcen weiterzuentwickeln (Teece 2014, 338 f.).

Trotz der unterschiedlichen Betrachtungsebenen (einzelner Akteur versus Unternehmen) bestehen erhebliche Zusammenhänge zwischen beiden Diskussionen. Dies beginnt bei der Terminologie, schließt aber auch den Ergebnisbezug ein. Auch hier handelt es sich um Dispositionen, mit denen etwas Angestrebtes erreicht werden soll. Werte, Normen und Kultur bilden ordnende Momente bei der Anwendung. Man kann sogar einen Zusammenhang über verschiedene Betrachtungsebenen unterstellen: Damit es zur Entwicklung von Kernkompetenzen kommt, bedarf es der Realisierung von individuellen Kompetenzen. ${ }^{11}$ Haben sich diese Kernkompetenzen einmal in der Organisation verfestigt, leiten sie an, welche individuellen Kompetenzen besonders gefragt sind (Meynhardt 2007, 312). Ein wesentlicher Unterschied zwischen beiden Betrachtungsebenen ist allerdings, dass eine Kernkompetenz im Allgemeinen nicht von einer einzelnen Person verwirklicht wird. Vielmehr emergiert sie aus dem Zusammenwirken vieler Personen. Das heißt, Kernkompetenzen müssen von einzelnen Individuen loslösbar sein. ${ }^{12}$ Dies bedeutet aber auch, dass manche Individualkompetenzen erst im Team zur Entfaltung kommen können. So besteht eine Abhängigkeit voneinander, die sich aus dem synergetischen Zusammenspiel von individueller Mikro- und kollektiver Makroebene ergibt (Meynhardt 2007). Über organisatorische Lern- und Integrationsprozesse muss eine Auswahl und Integration stattfinden. Der Wettbewerbsvorteil entsteht dann auch aus der Einzigartigkeit der Kombination.

Fassen wir zusammen, dann können unter einer strategischen Führungskompetenz jene Teilkompetenzen verstanden werden, die von den einzelnen strategischen Führungskräften benötigt werden, um die wertgenerierenden, strategischen und zielorientierten Tätigkeiten einer Organisation zukunftsorientiert zu gestalten sowie möglichst viele Mitglieder der Organisation von den damit verbundenen Zielen und dem korrespondierenden Prozess der Zielerreichung zu überzeugen. Wo diese Kompetenz nicht vorhanden ist, erwächst ein nachhaltiger Wettbewerbsnachteil. Wo sie zum Beispiel auf einer Corporate-Ebene vorhanden ist, entstehen vertikale Managementsynergien, das heißt eine Ressource, die den zu führenden operativen Einheiten als Vorteil weitergegeben werden kann.

Versuchen wir nun, diese strategische Führungskompetenz in ihrer Spezifität näher zu erfassen, dann ist auf einige Herausforderungen hinzuweisen:

- Verständnis von Performance: Eine strategische Führungskraft erfüllt dann die Erwartungen, wenn ihre erbrachte Leistung gesamthaft aus Sicht der Organisation als wünschenswert und positiv im jeweiligen Kontext betrachtet werden kann. Doch diese Performance lässt sich aufgrund der gegebenen Komplexität und Vieldeutigkeit nur schwer bestimmen und zuordnen. Sie ist ein abstraktes Konstrukt, das physisch nicht abgreifbar ist. Deshalb muss man sich geeigneter Indikatoren, insbesondere Verhaltensmerkmale, für die Performance bedienen.

- Ursache-Wirkungs-Zusammenhangs: Die Grundannahme ist hier, dass Kompetenzen Mittel sind, durch deren Einsatz Performance erzielt wird. Deshalb misst man Kompetenzen, also Verhaltensdispositionen, eine hohe Vorhersagequalität bei: Ist die Kompe-

11 Rothaermel/Hess (2007, S. 915) haben diesbezüglich eine Heterogenität von Firmen hinsichtlich der Fähigkeit zur Innovation nachgewiesen.

12 Hamel (1994), S. 11 f.: „Core competence represents the integration of a variety of individual skills. It is this integration that is the distinguishing hallmark of a core competence. Thus a core competence is very unlikely to reside, in its entity, in a single individual or a small team." 
Bekemeier/Meynhardt/Müller-Stewens | Was von Entscheidungsträgern erwartet wird

tenz vorhanden und wird sie auch mobilisiert, so kann mit einer hohen Performance gerechnet werden. ${ }^{13}$ Doch dieser unterstellte Ursache-Wirkungs-Zusammenhangist schwer messbar und kann sich über die Zeit ändern. ${ }^{14}$ Und natürlich ist es nicht nur die Kompetenz, die die Performance bestimmt, vielmehr spielen viele andere Faktoren wie etwa Job-Autonomie, Ressourcenverfügbarkeit oder Karriereaussichten hier mit herein.

- Kontextabhängigkeit: Es ist direkt plausibel, dass der Arbeitskontext (Branche, Land, Funktion, Situation des Unternehmens usw.) eine große Rolle für Kompetenzen spielt. ${ }^{15}$ Das heißt, dass das Verhalten eines strategischen Entscheidungsträgers eine Funktion seiner selbst und der Situation, in der er sich befindet, ist. Dies gilt umso mehr, je höher die hierarchische Position der Person ist (Hoffmann et al. 2011, 353). Diese Einsicht hat jedoch Konsequenzen bezüglich der Generalisierbarkeit eines zu entwickelnden Kompetenzmodells: Für welche Firmen, Situationen und Hierarchieebenen gelten die Kompetenzen eines spezifischen Modells? Auch verlangen unterschiedliche Strategien unterschiedliche Kompetenzen (Thomas/Ramaswamy 1996). Ein Modell muss daher trotz des Strebens nach Generalisierbarkeit in der Lage sein, den Kontext zu berücksichtigen. Dies kann zum Beispiel über das Setting der Teilkompetenzen geschehen oder über deren jeweils als notwendig erachtete Ausprägung.

- Wechselseitige Abhängigkeiten: Es ist davon auszugehen, dass Abhängigkeiten zwischen Teilkompetenzen bestehen. Das heißt, je nach Problemstellung kommt es zu einem unterschiedlichen Zusammenspiel der Teilkompetenzen (Catano/Darr/Campbell 2007).

- Stärken-Schwächen-Paradoxon: Eine alte Frage lautet: Können aus Stärken Schwächen werden? Im Kontext dieses Forschungsprojektes bedeutet dies: Ist eine höhere Ausprägung einer spezifischen Kompetenz immer besser oder gibt es auch eine zu starke Ausprägung, ein Zuviel? Können extreme Kompetenzausprägungen zu einer Schwäche werden?Die Antwort ist: ja. Dafür liegen inzwischen diverse empirische Belege vor. Deshalb gehen wir hier vom Stärken-Schwächen-Paradoxon aus, das uns eine realistischere Betrachtung der Wirklichkeit ermöglicht als die Annahme von rein linearen Beziehungen. ${ }^{16}$

\subsection{Von der Kompetenz zum Kompetenzmodell}

Wenn hier gesagt wird, dass ein Modell zur strategischen Führungskompetenz entwickelt wird, dann ist damit eine Sammlung und Beschreibung von Teilkompetenzen gemeint, die als Voraussetzung dafür erachtet werden, dass eine Führungskraft in einer gegebenen inund externen Situation strategisch erfolgreich agiert. ${ }^{17}$ Dabei geht es uns aber nicht nur

13 Ramo/Saris/Boyatzis (2009) weisen dies zum Beispiel nach. So konnte gezeigt werden, dass sich die „top-level executive performance“ zuverlässig durch die Erhebung der „top-level executive competencies“ vorhersagen lässt (Russell 2001, S. 568). Manche meinen sogar zeigen zu können, dass „aggregated managerial competencies are positively related to unit performance" (Levenson/Van der Stede/ Cohen 2006, S. 364).

14 Vgl. zu dieser Beobachtung Russell (2001) und Molleman/van der Vegt (2007), S. 417.

15 Thomas/Ramaswamy (1996) weisen dies zum Beispiel auch empirisch nach.

16 Die entsprechende Diskussion wird im Folgenden nach Erich Fromm als Stärken-Schwächen-Paradoxon bezeichnet: „Es beruht auf dem Gedanken, dass unsere Schwächen oft wenig mehr sind als der übertriebene Einsatz unserer Stärken“ (Rarrek/Werner 2012, S. 47). Laut Fromm liegt der Schlüssel zu produktiverem Verhalten unter anderem darin, die Überbeanspruchung und den übertriebenen Einsatz von Stärken zu begrenzen.

17 Vgl. ähnlich Krumm/Mertin/Dries (2012). Mit der Erarbeitung von Kompetenzmodellen kommt man natürlich auch mit der Leadership-Forschung, der sogenannten Eigenschaften- bzw. Trait-Forschung, 
um die Darstellung des Kanons an Teilkompetenzen im Modell, sondern auch um dessen Operationalisierung.

\section{(1) Kompetenzkategorien und Teilkompetenzen}

Eine erste Frage, die sich bei der Erarbeitung eines Kompetenzmodells stellt, ist, ob es gewisse Grundkategorien an Kompetenzen gibt, auf denen ein solches Modell aufbauen kann, um dann auf einer nächsten Ebene spezifiziert zu werden. In der Literatur trifft man häufig auf folgende pragmatische Dreiteilung, die sich vielerorts bewährt hat: ${ }^{18}$ (1) die Selbstkompetenz, (2) die Fachkompetenz sowie (3) die Sozialkompetenz. Manche Autoren ergänzen diese drei Grundkompetenzen noch um eine vierte, eine aktions- und umsetzungsorientierte Kompetenz (Erpenbeck/von Rosenstiel 200, Meynhardt 2004, Wundererl Bruch 2000).

Es geht hier um den Ergebnisbezug des Handelnden, seine Kompetenz, Handlungen zu initiieren, durchzuführen und hinsichtlich ihres Ergebnisses zu evaluieren. Die Forderung nach der vierten Grundkompetenz wird dadurch legitimiert, dass Handlungen bei gleichem Gegenstandsbezug des Handelnden zum Beispiel aufgrund von Engagement oder Hartnäckigkeit in ihrer Umsetzungsqualität variieren können.

Auf einer zweiten Ebene werden solche Kompetenzkategorien über Teilkompetenzen spezifiziert. Diese sind es dann auch, die beurteilt werden. Dabei sind die einzelnen Teilkompetenzen nicht unabhängig voneinander. Sie sind interaktiv und es gibt Austauschbeziehungen. Das heißt, effektive Führung ist mehr als nur die Ausprägung aller Teilkompetenzen. Auch ist die Konsistenz der zur Anwendung kommenden Teilkompetenzen bedeutsam.

In der Unternehmenspraxis hat die Anwendung von Kompetenzmodellen eine lange Tradition. ${ }^{19}$ Ein Vorteil gut fundierter Kompetenzmodelle ist, dass sie eine ganzheitlichere Personalarbeit von der Rekrutierung bis zur Mitarbeiterentwicklung ermöglichen. ${ }^{20}$ Sie bieten die Möglichkeit, Strategien in einer Organisation zu verankern, sie zu operationalisieren (Sanchez/Levine 2009). Damit bilden sie für die Mitarbeiter eine Brücke zwischen deren Selbstmanagement und der oft abstrakten Strategie. Aufgrund ihrer Standardisierung helfen sie auch, Vergleiche durchzuführen.

Eine Anforderung an das zu entwickelnde Kompetenzmodell ist, dass es an den Kontext, die betrachtete Position und die Strategie des anwendenden Unternehmens anpassbar ist und damit auch Zukunftsbezug hat.

Zusammenfassend lässt sich sagen, dass ein sorgsam entwickeltes Kompetenzmodell, bei dessen Entwicklung die kritische Diskussion zu Kompetenzmodellen aufgegriffen wurde,

in Berührung. Hierzu gibt es viel Kritik, insbesondere was simple, monokausale Modelle anbelangt. So fordert zum Beispiel Zaccaro (2007), dass ganzheitliche, multidimensionale Konstrukte entwickelt werden sollten. Doch auch hier stellen zum Beispiel Hoffmann/Woehr/Maldagen-Youngjohn/Lyons (2011) den Nachweis eines Zusammenhangs mit der Performance infrage.

18 Vgl. zum Beispiel Erpenbeck (2012), S. 16. Er unterteilt in personale, fachlich-methodische und sozialkommunikative Kompetenzen. Diese Dreiteilung baut auf der Dreiweltentheorie von Popper (2004, S. 84 ff.) und der Theorie des kommunikativen Handelns von Habermas (1995) auf.

19 Vgl. zum Beispiel das unternehmensspezifische Modell von 3M bei Allredge/Nilan (2000) oder das mit der SHL Group entwickelte, unternehmensübergreifende Modell von Kurz/Bartram (2002).

20 Vgl. zum Einfluss der Personalarbeit auf die Performance von Unternehmen vgl. Sonntag/Stegmaier (2006), S. 391. 
Bekemeier/Meynhardt/Müller-Stewens | Was von Entscheidungsträgern erwartet wird

als solide Grundlage für ein Personalmanagement betrachtet werden kann. ${ }^{21}$ Entsprechend erfreuen sie sich sowohl in der Forschung als auch in der Unternehmenspraxis einer regen Aufmerksamkeit.

\section{(2) Kritik an Kompetenzmodellen}

Einer der wesentlichen Kritikpunkte an Kompetenzmodellen ist, dass unklar und beliebig bleibt, was genau unter einer Kompetenz zu verstehen ist und wie sie von anderen Konstrukten abzugrenzen ist. So gibt es immer noch keine einheitliche Definition dafür, was unter einer Kompetenz zu verstehen ist. Diese Kritik trifft wohl zu, wenn eine Kompetenz als ein psychologisches Konstrukt, zum Beispiel für die Persönlichkeit von jemandem, verstanden wird. Doch das hier angewandte holistische Kompetenzverständnis schließt eine solche Deutung aus. Natürlich geht mit dem hier vorgestellten Ansatz auch eine gewisse Subjektivität einher. Doch wird in jedem gewählten Ansatz so oder so von einer subjektiven, sozial konstruierten Realität ausgegangen.

Weiter wird kritisiert, dass Kompetenzmodelle zu generisch seien und damit das Potenzial solcher Modelle überschätzt werde. Im dem hier entwickelten Modell wird zwar mit solchen generischen Kompetenzkategorien und Teilkompetenzen gearbeitet, sie werden aber je nach Kontext und Situation angepasst. Als Folge dieses Kritikpunkts lässt sich übrigens beobachten, dass man sich generell mehr und mehr interpretativen und situativen Ansätzen zuwendet; interpretativ in dem Sinne, dass das, was eine erforderliche Kompetenz ist, meist durch die Wahrnehmung des Individuums geprägt und konstituiert wird. Auch die situativen Ansätze beziehen den in- und externen Handlungskontext ein.

Einem anderen Kritikpunkt zufolge fehlt es an ausreichend validen empirischen Belegen dafür, dass Kompetenzen einen positiven Zusammenhang zum beruflichen Erfolg haben. Auch wird kritisiert, dass die Erhebung der Kompetenzen - zum Beispiel mittels „Critical Incident Interviews“ - problematisch sei, wenn es darum gehe, herauszubekommen, was die Befragten tun, denken oder fühlen.

Natürlich sind wir uns bei der Herleitung des Modells bewusst, dass Führungskräfte nicht über einen Kamm geschoren werden können und dass es nicht nur einen Weg gibt, um eine strategische Problemstellung erfolgreich zu bearbeiten. Auch ist es wohl kaum möglich, ein Modell und ein dazugehöriges Anwendungsverfahren zu entwickeln, das alle bislang diskutierten Teilaspekte abdeckt (Tett/Burnett 2003, 511), und es wird wohl nie eine „beste“ Taxonomie von Teilkompetenzen oder Performance-Dimensionen geben. Trotzdem wollen wir hier mit einem möglichst konkreten Verständnis einer strategischen Führungskompetenz arbeiten, um das gesetzte Ziel zu erreichen. Dabei nehmen wir keine Allgemeingültigkeit für uns in Anspruch, meinen aber, dass ein solches Vorgehen im Rahmen der eigenen Zielsetzung tauglich ist. ${ }^{22}$

\section{Das Modell der Strategischen Führungskompetenzen zur Bewertung von Strategen}

Aufbauend auf den obigen Erkenntnissen aus der Kompetenzforschung gehen wir nun davon aus, dass es der beste Weg zu einem vertieften Verständnis der Performance von Führungskräften ist, sie dabei zu beobachten, was sie tun, um erfolgreich zu sein. Sie sollten in

21 Zu diesem Schluss kommt auch Sonntag (2006).

22 Diese Art der Legitimation findet sich auch bei Borman/Brush (1993), S. 19.

Die Unternehmung, 74. Jg., 1/2020 
Situationen beobachtet werden, in denen möglichst viele Aspekte der gesuchten Kompetenz zur strategischen Führung zum Vorschein kommen. ${ }^{23}$

Um dies leisten zu können, wurde das Modell der Strategischen Führungskompetenzen (MSF) entwickelt. Es ist ein konzeptionelles Instrument, das als Hilfestellung bei der Betrachtung einer strategischen Führungskompetenz dienen soll, ohne Anspruch auf Vollständigkeit oder Absolutheit zu erheben. So gibt es viele andere Erklärungsansätze für eine gegebene Realität. Unser Modell stellt lediglich einen fundierten Ansatz dar, darzustellen, wie die Erfahrungen und das Wissen von Führungskräften zum Nutzen der Organisation in einer systematischen und wohlbegründeten Art und Weise eingesetzt werden.

Die Herleitung des Modells erfolgte über einen neunstufigen Forschungsprozess. Ziel dieses Prozesses war es zum einen, methodisch möglichst sauber und fundiert Wissen zu dem Konstrukt einer strategischen Führungskompetenz zu generieren. Zum anderen sollte dieses Wissen für die Unternehmenspraxis nutzbar und anschlussfähig sein. Um dieses Wissen zu generieren, war es notwendig, strategische Führungskräfte in ihrem sozialen beruflichen Umfeld zu beobachten, zu analysieren und zu interpretieren. ${ }^{24}$ Dazu wurde wie folgt vorgegangen, wobei die Vorgehensweise hier nur grob umrissen werden kann: ${ }^{25}$

(1) Interviews zur thematischen Einstimmung: Zur Bildung eines Gespürs und ersten Verständnisses für die Thematik und zur Annäherung an das Konstrukt Strategische Führungskompetenz (SFK)wurden acht explorative, narrative und semi-strukturierte Leitfadeninterviews mit erfahrenen Partnern der Executive-Search-Firma Boyden aus Amerika, Asien und Europa geführt. Diese Art von Interviews sind für komplexe und offene Fragen - wie im vorliegenden Fall - besonders geeignet. Sie basieren auf den Erfahrungen der Interviewten, die in Form von Erzählungen wiedergegeben werden. Neben Fragen zum persönlichen und organisatorischen Kontext wurde auch gefragt, welche Rolle bislang das Thema Strategiekompetenz im Prozess der Suche nach geeigneten Kandidaten für Führungspositionen in einem Unternehmen spielt, was der Interviewte damit assoziiert, wie er sie bislang evaluiert und was für ihn einen erfolgreichen Strategen ausmacht.

(2) Festlegung der Stichprobe für die Experteninterviews: Als Nächstes musste die Stichprobe für Experteninterviews zur Erforschung des Konstrukts SFK definiert werden. Zielgruppe waren ober(st)e strategische Führungskräfte, die ihr Unternehmen gut, erfolgreich und besonders zukunftsorientiert leiten.

Hinsichtlich der Stichprobengröße sind wir nach Maßgabe der Forschungsmethodik der "Grounded Theory" vorgegangen (Glaser/Strauss 1999; Strauss/Corbin 1998; Charmaz 2006): Man erhebt so lange Daten, bis eine Sättigung eintritt, das heißt, zusätzliche Interviews kaum noch wesentliche neue Erkenntnisse bringen. Maßstab dafür ist, dass parallel zur Datensammlung schrittweise eine Theorie entwickelt wird, die in die Interviewführung zurückgespielt wird.

Konkret wurden 28 auswertbare Interviews durchgeführt. Dabei war das Sample der Interviewten sehr divers, was eine breite Einsicht ermöglicht. 20 der Interviewten sind schwerpunktmäßig in Westeuropa tätig, 5 in Afrika, 2 in Australien und einer in Osteuropa. Sie sind in 12 verschiedenen Branchen tätig, im Schnitt gut 12 Jahre im Unternehmen und seit knapp 6 Jahren in der jeweiligen Position. Alle Interviewten gehörten der ersten Managementebene an, waren also auch strategische Entscheider.

23 Vgl. hierzu auch Athey/Orth (1999).

$24 \mathrm{Zu}$ einer detaillierten Darstellung des Forschungsprozesses vgl. Bekemeier (2016), Kapitel 3.

$25 \mathrm{Zu}$ einer detaillierten Darstellung des Forschungsprozesses vgl. Bekemeier (2016), Kapitel 3. 
Bekemeier/Meynhardt/Müller-Stewens | Was von Entscheidungsträgern erwartet wird

(3) Auswabl der Interviewer: Die Interviewer waren Partner des Projektpartners, der Executive-Search-Firma Boyden. Sie sind deshalb besonders geeignet, da Kompetenzbeurteilungen ihr tägliches Brot sind und sie auch mit derartigen Interviewtechniken vertraut sind. Alle brachten eine langjährige Erfahrung in diesem Berufsfeld mit.

(4) Durchführung der Interviews: Im Zentrum des Interviews stand eine spezielle Interviewtechnik: das „Behavioral Event Interview“, das auf der „Critical Incident Technique" 26 aufbaut. Über sie kann direkt erfasst werden, was Führungskräfte tun, um ihre Funktion im Unternehmen erfolgreich auszuüben. Der Interviewte soll hier ein für ihn sehr wichtiges und noch nicht allzu lange zurückliegendes Ereignis aus seiner individuellen Lebenswelt im Zusammenhang mit einer strategischen Entscheidung, in die er aktiv involviert war, möglichst präzise beschreiben: Was war für ihn die spezifische Ausgangslage? Wie hat er sich konkret verhalten? Was hat er konkret gedacht und gefühlt? Was war exakt das Ergebnis? Dies sind zwar retrospektive Erzählungen, doch die besten Vorhersagen hinsichtlich zukünftiger Performance erlangt man über das Verstehen vergangener Performance. ${ }^{27}$ Das heißt, wir gehen davon aus, dass aus vergangenen Verhaltensmustern und deren Ergebnissen recht gut auf zukünftige geschlossen werden kann. Im vorliegenden Projekt wurden die Befragten nach drei solchen für sie bedeutsamen Ereignissen gefragt, bei einer durchschnittlichen Interviewdauer von einer Stunde. Eines der Ereignisse sollte einen positiven Ausgang gehabt haben, ein zweites einen negativen und ein drittes sollte mit einem besonders hohen Maß an Unsicherheit verbunden gewesen sein.

Auch für diese Interviews wurde ein Leitfaden für die Interviewer erstellt, der den gesamten Interviewprozess abbildete. Der Leitfaden sorgte für eine gewisse Standardisierung der Interviews zur Sicherstellung des Forschungsinteresses und der Datenqualität. Auch wurde damit die Vergleichbarkeit der Daten erhöht. Zuerst wurde in das Interview und seine Absicht eingeführt, dann ging es um die konkrete Position und Verantwortung des Interviewten, dann um die drei „Behavioral Events“ und letztlich um die Frage, was es braucht, um die Aufgabe wirksam zu bewältigen. ${ }^{28}$ Die Erkenntnisse für das Modell der strategischen Führung stammen schwerpunktmässig aus den verhaltensbezogenen Fragen im Hauptteil des Leitfadens. Selbiger wurde vorgängig getestet und auf der Basis der in den Interviews gewonnenen Erfahrungen immer wieder weiterentwickelt.

(5) Bildung von Kontrastgruppen: Zur Differenzierung von Führungskräften mit sehr erfolgreichen und durchschnittlich erfolgreichen Verhaltensweisen wurde das Sample auf der Basis einer mehrdimensionalen Performance-Beurteilung in zwei Gruppen geteilt. Diese Beurteilung erfolgte durch den Interviewer nach der Durchführung des Interviews.

Da die individuelle Leistung, wie zuvor ausgeführt, nicht direkt beobachtbar ist, braucht es Ergebniskriterien als stellvertretende Proxys. Hier wurden vier als geeignet erachtet: (a) Kriterien zur finanziellen Performance der Organisation (zum Beispiel ROI oder Umsatzwachstum), (b) Kriterien zur nicht finanziellen Performance (zum Beispiel Kundenzufriedenheit oder Produktqualität), (c) die Konstanz der individuellen Karriere sowie (d) eine Gesamteinschätzung. Jeder Proxy musste auf einer Skala von 1 (=poor) bis 10 (=excellent) bezogen auf den Interviewten evaluiert werden. Die vier Proxys wurden gleich gewichtet. Die Interviewer sind trainierte Beurteiler, insofern war das Risiko von Urteilsverzerrungen eher gering. Da sich in der Stichprobe so oder so nur erfolgreiche Führungskräfte befan-

26 Diese Technik wurde von Flanagan (1954) entwickelt.

27 Vgl. dazu zum Beispiel Zwell (2000), S. 255.

28 Man orientierte sich dabei an Spencer/Spencer (1993), S. 119.

Die Unternehmung, 74. Jg., 1/2020 
den, wurde der Schnitt bei einer relativ hohen Ausprägung gesetzt: Alle mit einem Performance-Score größer als 8 wurden als Top-Performer eingeteilt. Von den 28 Interviewten waren dies 12.

(6) Codierung der Daten: Interviewführung und Datenanalyse erfolgten personell getrennt. So wurde zum einen eine globale Datenerhebung durch die internationalen Boyden-Partner möglich, zum anderen war die Auswertung durch das Forschungsteam damit weitgehend frei von persönlichen Vorurteilen der Interviewer, die eventuell im Interview entstanden sind.

Die Datenauswertung erfolgte weitgehend ergebnisoffen. So wurde bewusst nicht auf bestehende Checklisten von Kompetenzen zurückgegriffen, da es ja darum ging, auch offen für neue Kompetenzen, die den erfolgreichen Strategen ausmachen, zu sein. ${ }^{29}$

Zur Datenauswertung wurden die Interviews transkribiert und das in den Interviews gezeigte Verhalten hinsichtlich erkennbarer Kompetenzen kodiert. Dabei folgte man wiederum dem methodischen Rahmen der Grounded Theory: Induktiv werden hier aus den Daten Konzepte und Theorien in einem kontinuierlichen Prozess hergeleitet. Es werden vorläufige Theorien aus den initialen Interviews entwickelt, die über nachfolgende Interviews hinterfragt und verfeinert werden.

In der Analyse werden Textpassagen Codes zugewiesen. Im vorliegenden Fall liefern die Codes die demonstrierten Selbstorganisationsdispositionen, die Kompetenzen, die man als Interviewer in die gezeigten Verhaltensweisen hineininterpretiert. Verbindungsstück zwischen den Daten und den Codes waren sogenannte Memos, in denen Ideen zur Theorieund Konstruktentwicklung entlang des Auswertungsprozesses festhalten wurden.

Die Ergebnisse des Analyseprozesses wurden dann in Form von Beziehungsnetzwerken und Baumdiagrammen visualisiert. Zur Sicherstellung der Intersubjektivität wurde auch eine zweite Codierung durch einen nicht in das Projekt eingebundenen Forscher durchgeführt. Am Ende ergab dies ein Kodierschema von 57 Codes.

(7) Abgleich der Kontrastgruppen: Im abschließenden Schritt der Datenanalyse galt es, die Unterschiede zwischen den Durchschnitts- und Top-Performern herauszuarbeiten. Um eine bessere Übersicht über die große Datenmenge zu erhalten, wurde das ganze Material in sechs thematische Cluster (zum Beispiel „Empowering“ oder „Execution“) aufgeteilt und dort der Abgleich durchgeführt.

Dabei zeigte sich zum Beispiel, dass die Top-Performer deutlich homogenere Verhaltensmuster zeigen. Auch zeigen sie einen breiteren Blickwinkel auf die Situation und sehen eher das Big Picture. Ihre Denkweise ist dynamisch-prozessorientiert. Sie fokussieren mehr auf die Kunden als auf die Märkte. Auch wird die Bedeutung der Selbstreflexion mehr hervorgehoben: zum Beispiel bei Entscheidungen auch einmal einen Schritt zurück zu machen, um sich mehr Zeit für die Entscheidung zu nehmen. Immer wird bei ihnen das Management von Spannungen und Dilemmata angesprochen und die Notwendigkeit, diese produktiv und lösungsorientiert zu handhaben. Sie sehen sich eher als Befähiger derer, an die sie Arbeit delegieren, und als Vermittler. Zudem stehen bei ihnen die fachlichen Aspekte nicht so stark im Vordergrund wie bei den Durchschnitts-Performern.

(8) Herleitung einer Liste potenzieller Teilkompetenzen aus der Theorie: Um ein Verständnis für die möglichen Inhalte des Konstrukts SFK zu entwickeln, wurde nach beste-

29 Aber man war sich natürlich der Tatsache bewusst, dass ein solcher Prozess schon allein wegen der Kenntnis der Literatur zur Kompetenzforschung nie gänzlich ergebnisoffen sein kann. 
Bekemeier/Meynhardt/Müller-Stewens | Was von Entscheidungsträgern erwartet wird

henden theoretischen Erkenntnissen gesucht, die auch als Input für das MSF dienen sollten. Dazu wurden drei Arten von Quellen herangezogen: Managementklassiker, existierende Kompetenzmodelle und -taxonomien sowie spezifische Fachliteratur und Experteneinschätzungen. Die Ergebnisse wurden mittels Fokusgruppen zu einer umfassenden Liste potenzieller Teilkompetenzen integriert. ${ }^{30}$

(9) Herleitung des Modells der Strategischen Führungskompetenzen : Die Erkenntnisse aus der Datenanalyse der Behavioral Event Interviews und die explorativen Expertengespräche mit den Boyden-Partnern, die aus der Analyse der theoretischen Literatur zur strategischen Führung hergeleiteten Listen potenzieller Teilkompetenzen sowie die Erfahrungen und Kenntnisse der in den Prozess involvierten Experten bildeten die Inputs für die Herleitung des MSF. Hauptaufgabe war es nun,

(a) die relevanten Teilkompetenzen einer SFK zu identifizieren,

(b) diese präzise zu definieren und

(c) ihre möglichen Ausprägungsstufen über Verhaltensindikatoren, die aus den Situationsbeschreibungen der Interviewten gewonnen wurden, verbal zu verankern.

Die Erkenntnisse aus den Interviews mit Strategen, aus explorativen Expertengesprächen mit den Boyden-Partnern, aus den Listen potenzieller Teilkompetenzen, die auf der Analyse der theoretischen Literatur zur strategischen Führung basieren, sowie aus den Erfahrungen und Kenntnissen der in den Prozess involvierten Experten bildeten die Inputs für die Herleitung des MSF. Die Hauptaufgabe war es dann, (a) die relevanten Teilkompetenzen einer SFK zu identifizieren, (b) diese präzise zu definieren und (c) ihre möglichen Ausprägungsstufen über Verhaltensindikatoren, die aus den Situationsbeschreibungen der Interviewten gewonnen wurden, verbal zu verankern. Das dabei nach und nach emergierende MSF wurde mehrfach Praxistests unterzogen, indem es auf die erhobenen Daten angewandt wurde.

\subsection{Der Aufbau des MSF}

Mit dem MSF wird ein ausgeprägter Handlungsfokus eingenommen, dem ein komplexes holistisches Verständnis des Kompetenzkonstrukts als Selbstorganisationsdispositionen zugrunde liegt. In diesem Modell sollen jene Teilkompetenzen strategisch handelnder Führungskräfte repräsentiert sein, die als unabdingbar für den zukünftigen nachhaltigen Erfolg eines Unternehmens angesehen werden.

Die Entwicklung des MSF baut auf folgenden Annahmen auf:

- Das Konstrukt der individuellen Kompetenz zur strategischen Führung kann über ein Bündel geeigneter Teilkompetenzen angemessen erfasst werden. Diese Teilkompetenzen weisen bedeutsame Beziehungen eines Zusammenwirkens untereinander auf. Damit können sie aber auch nicht trennscharf voneinander abgegrenzt werden. Dieses Konstrukt soll andere Konstrukte (wie zum Beispiel Persönlichkeitskonstrukte aus der Psychologie) keinesfalls ersetzen, sondern sie ergänzen.

- Es besteht ein Zusammenhang zwischen der strategischen Führungskompetenz (mit ibren Teilkompetenzen) und der Performance des Unternehmens. Dieser Zusammenhang ist aber keineswegs als direkt und monokausal zu betrachten. Unter Performance

30 Vgl. zu dieser Liste Bekemeier (2016), S. 250-253. 
werden die positiven Ergebnisse individueller Handlungen in einem spezifischen Kontext verstanden.

- Aus dieser Kontextabhängigkeit der Performance ergibt sich die Kontextabhängigkeit der Teilkompetenzen. Trotzdem kann in dem MSF mit generischen Teilkompetenzen gearbeitet werden, denn die Anpassung an den jeweiligen Kontext und die Einordnung in die gegebene Situation erfolgt über deren Ausprägungen. Damit soll eine bestmögliche Balance zwischen zu generischen und zu spezifischen Kompetenzen gewährleistet werden. Dabei kann es auch ein Zuviel in der Ausprägung einer Kompetenz geben, das heißt eine übermäßige Ausprägung einer Kompetenz, die zu einer zurückgehenden Leistungserbringung führt.

- Die Teilkompetenzen verlangen nicht nur eine möglichst präzise Definition, es müssen auch Verhaltensindikatoren und unterschiedliche Ausprägungsgrade angegeben werden, um eine Operationalisierung und Arbeit mit dem Modell im Alltag zu ermöglichen.

- Inhärent ist dem MSF aufgrund des Strategiefokus seine Zukunftsbezogenheit. Bewertet wird damit also das Vorhandensein von Kompetenzen zur Handhabung zukünftiger strategischer Herausforderungen - auch wenn dies methodisch nur über die Rekonstruktion vergangener und gegenwärtiger Situationen geschehen kann. Mit diesem Strategiefokus wird auch die Brücke zwischen der Performance auf der Unternehmensebene und der Kompetenz auf der individuellen Ebene geschlagen.

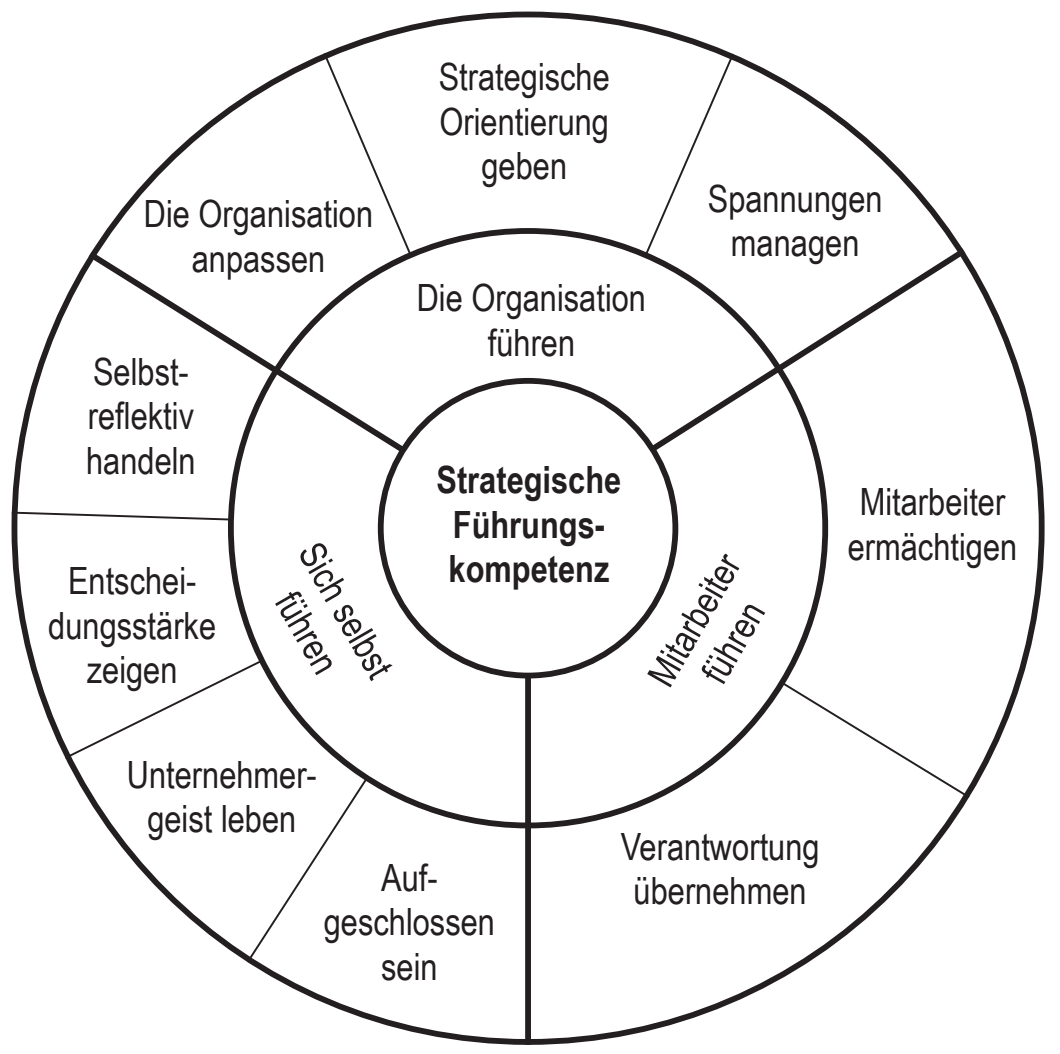

Abb. 1: Das Rad der strategischen Führungskompetenz 
Bekemeier/Meynhardt/Müller-Stewens | Was von Entscheidungsträgern erwartet wird

Im Folgenden sollen nun die einzelnen Elemente des in Abbildung 1 darstellten MSF - das „Rad der strategischen Führungskompetenz,, - hergeleitet und erläutert werden. Im Modell werden drei Kompetenzkategorien unterschieden. Jede dieser Kompetenzkategorien besteht wieder aus einzelnen Teilkompetenzen, neun insgesamt. Diese sind gleich gewichtet und stehen unhierarchisch nebeneinander. Sie sind nicht als strikt voneinander getrennt zu verstehen, vielmehr ist davon auszugehen, dass manche miteinander zusammenhängen und sich gegenseitig beeinflussen.

Für jede der neun Teilkompetenzen sind fünf Ausprägungsstufen über eine Skala festgelegt, wie in Abbildung 2 dargestellt. Die einzelnen Stufen werden - exemplarisch in Abbildung 3 für die Teilkompetenz "Strategische Orientierung geben“ - auf der Basis von Verhaltensindikatoren näher beschrieben (Erpenbeck 2012, 27f.) Das heißt in der Anwendung, dass die Beurteilenden auf der Skala die Ausprägung markieren, die nach ihrem subjektiven Empfinden am ehesten dem beschriebenen Verhalten bei der thematisierten strategischen Problemstellung entspricht. Ein solches Einstufungsverfahren ist auch unter Bezug auf den Beurteilten hilfreich, denn so kann relativ präzise erklärt werden, was die Entwicklung zu einer höheren Kompetenzstufe ausmacht, und es kann besprochen werden, wie dies geschehen soll und mit welcher Unterstützung dabei zu rechnen ist.

\begin{tabular}{|c|c|c|c|c|c|c|c|c|c|c|c|c|}
\hline \multicolumn{3}{|c|}{$\begin{array}{l}\text { Basic } \\
\text { Performer }\end{array}$} & \multicolumn{3}{|c|}{$\begin{array}{l}\text { Advanced } \\
\text { Performer }\end{array}$} & \multicolumn{3}{|c|}{$\begin{array}{l}\text { High } \\
\text { Performer }\end{array}$} & \multicolumn{3}{|c|}{$\begin{array}{l}\text { Out- } \\
\text { performer }\end{array}$} & $\begin{array}{l}\text { Over-the-top } \\
\text { Performer }\end{array}$ \\
\hline \multicolumn{3}{|c|}{ Stufe 1} & \multicolumn{3}{|c|}{ Stufe 2} & \multicolumn{3}{|c|}{ Stufe 3} & \multicolumn{3}{|c|}{ Stufe 4} & Stufe 5 \\
\hline 0 & 1 & + & - & 2 & + & - & 3 & + & - & 4 & + & 5 \\
\hline \multicolumn{3}{|c|}{$\begin{array}{l}\text { - Verhaltens- } \\
\text { indikatoren }\end{array}$} & \multicolumn{3}{|c|}{$\begin{array}{l}\text { - Verhaltens- } \\
\text { indikatoren }\end{array}$} & \multicolumn{3}{|c|}{$\begin{array}{l}\text { - Verhaltens- } \\
\text { indikatoren }\end{array}$} & \multicolumn{3}{|c|}{$\begin{array}{c}\text { - Verhaltens- } \\
\text { indikatoren }\end{array}$} & $\begin{array}{l}\text { - Verhaltens- } \\
\text { indikatoren }\end{array}$ \\
\hline
\end{tabular}

Abb. 2: Die Ausprägungsstufen einer Teilkompetenz

Die den einzelnen Ausprägungen der Skala zugeordneten Verhaltensindikatoren und -beispiele sollen helfen, die Ausprägungsstufen besser zu verstehen, sind aber nicht als Checkliste zu verstehen. Vor dem Hintergrund der angestrebten Praxistauglichkeit des MSF werden die Beschreibungen bewusst knapp, aber auch möglichst einheitlich in Länge und Form gehalten. Jeweils besonders kennzeichnende Merkmale sind durch Fettdruck hervorgehoben.

Bei der Entwicklung einer tauglichen Skala konnten wir uns der Erkenntnisse zu Prozessen der Kompetenzentwicklung bedienen. ${ }^{31}$ So wird für die für das MSF herzuleitende Skala von drei Hauptdimensionen einer Kompetenzentwicklung ausgegangen. Erstens ist dies die zunehmende individuelle Erfahrung auf dem Weg zu einem intuitiv geleiteten Problemlösungsmodus - was natürlich nicht heißen soll, dass dann alles nur noch intuitiv geschieht. Eine höhere Ausprägungsstufe wird durch einen Lernprozess erreicht, in dem die gewonnenen Erfahrungen in neues Wissen transformiert werden (Kolb 1984).

Zweitens ist dies die zunehmende Handlungskomplexität, die sich zum Beispiel aus dem Kontext, der Situation oder dem involvierten Personenkreis ergibt. Drittens ist dies die Performance, die allerdings bei einem Zuviel an Kompetenz abnimmt.

31 Vgl. zum Beispiel Dreyfus (2004). 
Die Skala haben wir in fünf Entwicklungsstufen eingeteilt: von der Basisstufe 1 bis zur Stufe 5, auf der es zu einer übermäßigen Ausprägung der Teilkompetenz kommt. Wir nehmen also an, dass es auch ein Zuviel einer Kompetenz geben kann, was bedeutet, dass dann aus einer Stärke eine Schwäche wird. ${ }^{32}$ Die Ausprägungen auf den einzelnen Stufen sind kumulativ zu verstehen, das heißt, jede Stufe schließt das beschriebene Verhalten auf den niedrigeren Stufen mit ein. Dies muss nicht heißen, dass jede der vorangegangenen Verhaltensweisen von einer zu beurteilenden Person auch gezeigt wird.

Um im praktischen Einsatz des Modells eine weitere Differenzierung zu ermöglichen, gibt es auf den ersten vier Stufen eine Binnendifferenzierung: (-) Es besteht eine Tendenz zur nächstniedrigeren Stufe; $(=)$ wird vollumfänglich erfüllt; $(+)$ es besteht eine Tendenz zur nächsthöheren Stufe. Zur weiteren Vereinfachung wurde jede Entwicklungsstufe mit einer Bezeichnung versehen: Stufe 1: Basic Performer; Stufe 2: Advanced Performer; Stufe 3: High Performer; Stufe 4: Outperformer; Stufe 5: Over-the-top Performer. Anzumerken ist hierbei, dass die Skala nicht von „schlecht“ bis „gut“ geht, sondern dass Stufe 2 und auch Stufe 1 je nach Kontext schon eine gute und ausreichende Performance bezeichnen können.

Bei der Beurteilung ist darauf zu achten, dass - anders als bei Schulnoten - die Ausprägung 1 bereits eine gewisse Grundkompetenz unterstellt. Eine 0 bedeutet, der Kandidat unterliegt einem Knock-out-Kriterium (Preselection).

Nun können wir uns den einzelnen Teilkompetenzen mit ihren Ausprägungsstufen zuwenden. Der Leser ist eingeladen, sich selbst mithilfe der Verhaltensindikatoren auf den neun Skalen zu verorten.

\subsection{Die Kompetenzkategorien}

Die drei Kompetenzkategorien im MSF lauten: (a) „Die Organisation führen“, (b) „Mitarbeiter führen“ sowie (c) „Sich selbst führen“.

\section{(1) Die Organisation führen}

Die Kompetenzkategorie „Die Organisation führen“ besteht aus drei Teilkompetenzen: „Strategische Orientierung geben“, „Anpassung der Organisation“ sowie „Spannungen managen".

Strategische Orientierung geben: In Abbildung 3 wird diese Teilkompetenz definiert und mit den Verhaltensindikatoren der fünf Entwicklungsstufen dargestellt. ${ }^{33}$ Hier geht es um die Kompetenz, der Organisation eine strategische Ausrichtung zu geben, indem eine strategische Grundeinstellung gefördert wird, Raum für strategische Diskussionen geschaffen wird, diese priorisiert werden und das Geschäftsmodell aktiv gestaltet wird.

32 Vgl. den Nachweis dazu bei Gebert/Boerner/Lanwehr (2003).

33 Für die anderen acht Teilkompetenzen erfolgt eine solche tabellarische Darstellung in der englischen Originalfassung in Müller-Stewens (2019), Anhang A3. 
Bekemeier/Meynhardt/Müller-Stewens | Was von Entscheidungsträgern erwartet wird

\begin{tabular}{|c|c|c|c|c|c|c|c|c|c|c|c|c|}
\hline \multicolumn{13}{|c|}{$\begin{array}{l}\text { Strategische Orientierung geben } \\
\text { Gibt strategische Orientierung durch die Förderung einer strategischen Denkweise, die Schaffung von Raum für } \\
\text { strategische Diskussionen und deren Priorisierung sowie die Gestaltung von Geschäftsmodellen }\end{array}$} \\
\hline \multicolumn{3}{|c|}{$\begin{array}{c}\text { Basic } \\
\text { Performer }\end{array}$} & \multicolumn{3}{|c|}{$\begin{array}{l}\text { Advanced } \\
\text { Performer }\end{array}$} & \multicolumn{3}{|c|}{$\begin{array}{l}\text { High } \\
\text { Performer }\end{array}$} & \multicolumn{3}{|c|}{$\begin{array}{c}\text { Out- } \\
\text { performer }\end{array}$} & $\begin{array}{l}\text { Over-the-top } \\
\text { Performer }\end{array}$ \\
\hline \multicolumn{3}{|c|}{ Stufe 1} & \multicolumn{3}{|c|}{ Stufe 2} & \multicolumn{3}{|c|}{ Stufe 3} & \multicolumn{3}{|c|}{ Stufe 4} & Stufe 5 \\
\hline 0 & 1 & + & - & 2 & + & - & 3 & + & - & 4 & + & 5 \\
\hline \multicolumn{3}{|c|}{$\begin{array}{l}\text { - Denkt in einer } \\
\text { zukunftsorientierten } \\
\text { Art und Weise } \\
\text { - Respektiert Mission, } \\
\text { Werte, Vision und } \\
\text { Ziele } \\
\text { - Nutzt seine Analyse } \\
\text { und seine Kenntnis } \\
\text { des Geschäfts als } \\
\text { Grundlage seiner } \\
\text { Strategiearbeit }\end{array}$} & \multicolumn{3}{|c|}{$\begin{array}{l}\text { - Versteht die } \\
\text { Denkweise der } \\
\text { Kunden und passt } \\
\text { die Strategie } \\
\text { entsprechend an } \\
\text { - Nimmt eine echte } \\
\text { globale Perspektive } \\
\text { ein } \\
\text { - Verfügt über eine } \\
\text { gute strategische } \\
\text { Vorausschau und } \\
\text { antizipiert Trends }\end{array}$} & \multicolumn{3}{|c|}{$\begin{array}{l}\text { - Priorisiert Strategien } \\
\text { und etabliert Strate } \\
\text { giearbeit als eine } \\
\text { Routine } \\
\text { - Fördert die Kunden- } \\
\text { orientierung und } \\
\text { Schaffung von } \\
\text { Nutzen für den } \\
\text { Kunden als eine } \\
\text { strategische } \\
\text { Priorität } \\
\text { - Gestaltet die } \\
\text { wichtigsten An- } \\
\text { spruchsgruppen } \\
\text { beziehungen } \\
\text { - Hat eine klare } \\
\text { Botschaft hinsichtlich } \\
\text { der strategischen } \\
\text { Entwicklung } \\
\text { - Zeigt ein tiefes Ver- } \\
\text { ständnis des strate } \\
\text { gischen Kontexts } \\
\text { und integriert die } \\
\text { Einsichten daraus in } \\
\text { die Strategieent } \\
\text { wicklung }\end{array}$} & \multicolumn{3}{|c|}{$\begin{array}{l}\text { - Schafft Raum und } \\
\text { einen Sinn für } \\
\text { Dringlichkeit für } \\
\text { strategische } \\
\text { Diskussionen } \\
\text { - Gestaltet und } \\
\text { transformiert ganze } \\
\text { Geschäftsmodelle } \\
\text { - Gestaltet die } \\
\text { Mission, Werte, } \\
\text { Vision und Ziele } \\
\text { aktiv mit und } \\
\text { inspiriert die ganze } \\
\text { Organisation damit } \\
\text { - (Re)Konfiguriert } \\
\text { aktiv das Portfolio } \\
\text { der Geschäfte } \\
\text { - Treibt unablässig } \\
\text { die Entwicklung der } \\
\text { Organisation }\end{array}$} & $\begin{array}{l}\text { • Fokussiert fast } \\
\text { ausschliesslich auf } \\
\text { die strategische } \\
\text { Denkweise, verliert } \\
\text { dadurch den } \\
\text { Kontakt zum } \\
\text { täglichen Geschäft } \\
\text { und der Umsetzung } \\
\text { - Nimmt eine zu } \\
\text { ausgeprägte "Big } \\
\text { Picture"-Sichtweise } \\
\text { ein; hat seine } \\
\text { Gedanken } \\
\text { woanders } \\
\text { - Überhäuft die } \\
\text { Organisation mit zu } \\
\text { viel visionärem } \\
\text { Wandel was zu } \\
\text { Aktivismus führt } \\
\text { - Blind für } \\
\text { opportunistische } \\
\text { Gelegenheiten }\end{array}$ \\
\hline
\end{tabular}

Abb. 3: Definition und Skala der Teilkompetenz „Strategische Orientierung geben“

Die einzelnen Entwicklungsstufen dieser Teilkompetenz sind wie folgt zu interpretieren:

- Stufe 1: Vom Basic Performer wird zumindest erwartet, dass er zukunftsorientiert denkt und ihm der strategische Rahmen des Unternehmens (Mission, Werte, Vision und Ziele) nicht nur bekannt ist, sondern dass er ihn auch respektiert. Er verfügt als Basis für seine strategische Arbeit über ein gutes fachlich-analytisches Verständnis des eigenen Geschäfts.

- Stufe 2: Der Advanced Performer ergänzt dies um einen ausgeprägten Kundenfokus. Er versteht den Kunden in seinen Bedürfnissen und richtet seine Strategie danach aus. Er denkt global und verfügt über einen strategischen Weitblick.

- Stufe 3: Beim High Performer kommt ein erweitertes Verständnis des strategischen Kontexts und der Führungskraft hinzu und er integriert dies in den Strategieprozess. So sorgt er dafür, dass strategische Aktivitäten routinemäßig stattfinden. Er gestaltet be- 
wusst und systematisch seine Beziehungen zu den wichtigsten Anspruchsgruppen. Dabei hat die Nutzengenerierung für den Kunden oberste Priorität. Insgesamt vermittelt er eine klare Botschaft zur strategischen Entwicklung der Organisation.

- Stufe 4: Der Outperformer schafft erheblich Raum für die Strategiearbeit in der Organisation. Er erzeugt bei den Mitarbeitern ein Verständnis für deren Bedeutung und Dringlichkeit. Er wirkt inspirierend auf die Strategieinhalte ein. So kann er zum Beispiel bestehende Geschäftsmodelle grundsätzlich hinterfragen und zu deren Innovation und Transformation anregen. Auch treibt er die kontinuierliche Weiterentwicklung des Portfolios der Geschäfte in Richtung einer zukunftstauglichen Zusammensetzung.

- Stufe 5: Wird dies übertrieben, dann sind wir beim Over-the-top Performer angelangt. Er bewegt sich primär nur noch auf der abstrakt-theoretischen Strategieebene und vernachlässigt die Umsetzung sträflich. Sein übertriebenes Denken in Big Pictures und Visionen verunsichert und überfordert die Mitarbeitenden. Mit seinem strategischen Aktionismus verpasst er auch sich ergebende Opportunitäten.

Anpassung der Organisation: Mit ihr wird die Teilkompetenz beschrieben, die Organisation an die Strategie anzupassen und damit die Strategie zielstrebig umzusetzen, wozu ein effizientes und effektives Management der Organisation gehört sowie beispielsweise die erfolgreiche Initiierung und das Management strategischer Initiativen. Die fünf Entwicklungsstufen stellen sich wie folgt dar:

- Stufe 1: Der Basic Performer plant die Umsetzung der Strategie immer mit. Er definiert hierfür klare Verantwortlichkeiten und hat ein gutes Gespür für die Anforderungen, denen die Organisation dabei gerecht werden muss.

- Stufe 2: Der Advanced Performer sorgt für effiziente Organisationsstrukturen und Managementsysteme. Er demonstriert Beharrlichkeit und Ausdauer in seiner strategischen Führung und weiß, wie man sowohl auf formellem als auch auf informellem Wege die strategischen Aufgaben umsetzen und erledigen kann.

- Stufe 3: Auch der High Performer ist in der Lage, generell Strukturen, Systeme und Prozesse so zu gestalten, dass sie die Strategie bestmöglich unterstützen. Dies ermöglicht ihm sein tiefes Verständnis des Gesamtkontexts. Zur Strategieumsetzung stößt er strategische Initiativen erfolgreich an. Es gelingt ihm, eine ausgeprägte Ergebnis- und Erfolgsorientierung in der Organisation zu etablieren.

- Stufe 4: Der Outperformer stößt strategische Initiativen nicht nur an, sondern überprüft ihren Fortschritt auch regelmäßig und nimmt, falls notwendig, Anpassungen vor. Er ist hinsichtlich der Strategieumsetzung nicht dogmatisch, sondern demonstriert durchaus Flexibilität beziehungsweise Resilienz. Er ist auch in der Lage, Ressourcen für die Strategieumsetzung zu mobilisieren. Er hat ein gutes Gefühl für Timing und ergreift Chancen und Gelegenheiten, wenn sie sich ihm bieten. Er etabliert eine starke, strategieorientierte Organisationskultur und stellt einen strategieorientierten Führungsstil in der Organisation sicher. So schafft er eine gemeinsame Identität und stellt eine gewisse Kontinuität in der Organisation und deren Strategiearbeit sicher.

- Stufe 5: Der Over-the-top Performer übertreibt dies, indem er sich beispielsweise deutlich zu stark auf Strukturen, Prozesse und Systeme sowie ihre Anpassung an die Strategie konzentriert und dabei die Menschen und andere wichtige Unternehmensaspekte aus den Augen verliert. Die Anpassung der Organisation wird mit zu viel Druck zur fal- 
Bekemeier/Meynhardt/Müller-Stewens | Was von Entscheidungsträgern erwartet wird

schen Zeit durchgeführt. Perfektionismus im Rahmen dieser Anpassungen ist bei ihm wichtiger geworden als pragmatisches Arbeiten.

Spannungen managen: Diese Teilkompetenz beinhaltet, regelmäßig mit unternehmerischen Dilemmata umgehen zu können. Dazu gehört erstens, zunächst einmal die Existenz diverser Paradoxien überhaupt zu akzeptieren; zweitens gilt es, abstrakt und ganzheitlich denken zu können, um in einer komplexen Realität erfolgreich zu führen. Die fünf Entwicklungsstufen stellen sich wie folgt dar:

- Stufe 1: Der Basic Performer nimmt die Existenz einer Vielzahl von Dilemmata sowie diverser Trade-offs wahr, beispielsweise das Spannungsverhältnis zwischen kurzfristigen Ergebniszielen und langfristiger Strategie oder zwischen dem Management des Corporate- und jenem des Business-Unit-Levels. Dabei denkt er nicht mehr in reinen SchwarzWeiß-Mustern. Er akzeptiert auch die Vernetzung von unternehmerischen Handlungen und Entscheidungen.

- Stufe 2: Beim Advanced Performer kommt u. a. die Fähigkeit hinzu, eine Vielzahl von Informationen zu verarbeiten sowie verschiedene Interessen wahrzunehmen und damit umzugehen. Dies ist eine Voraussetzung dafür, komplexe Situationen schnell und rechtzeitig zu durchschauen. Er nimmt sich der Konflikte und Spannungsfelder an und demonstriert die Bereitschaft, mit ihnen umzugehen beziehungsweise sie zu managen.

- Stufe 3: Der High Performer widmet sich dem Management einer ganzen Reihe an Konflikten auf diversen Ebenen und über Organisationsgrenzen hinweg. Er findet bei Konflikten oft Gemeinsamkeiten, was ihm ein lösungsorientiertes Arbeiten ermöglicht. Er ist in der Lage, Konflikte und Dilemmata auszuhalten und in gleichem Zuge zu versuchen, in konfliktären Situationen das Optimum zu erreichen. Es gelingt ihm, Konflikte als Chancen zu formulieren und aktiv zu nutzen.

- Stufe 4: Der Outperformer ist in der Lage, effektiv mit unternehmerischen Dilemmata umzugehen und diese aktiv zu managen. Er ist fähig, in komplexen Paradoxien zu denken, und übernimmt dabei eine ausgleichende Rolle innerhalb der Organisation. Insbesondere verfügt er über die Kompetenz, scheinbare Gegensätze und Widersprüche neu in Bezug zueinander zu setzen und sie aus einer anderen Perspektive zu betrachten, um letztlich ein Ziel zu erreichen.

- Stufe 5: Der Over-the-top Performer übertreibt diese Kompetenz, indem er alles als Dilemma betrachtet und problematisiert. Er fokussiert sich nur noch auf reale und mutmaßliche Dilemmata und deren Lösungen, verliert sich dabei in diesen Tätigkeiten und kann den Blick für das große Ganze nicht bewahren. Er vermeidet Entscheidungen und versucht, Probleme auszusitzen. Kompromisse werden wichtiger als alles andere, sodass er letztlich den Eindruck vermittelt, keine eigene Meinung zu haben.

\section{(2) Mitarbeiter führen}

Die Kompetenzkategorie „Mitarbeiter führen“ besteht aus zwei Teilkompetenzen: „Verantwortung übernehmen“ und „Mitarbeiter ermächtigen“.

Verantwortung übernehmen: Diese Teilkompetenz umfasst das Demonstrieren von verantwortungsvoller Führung. Dazu gehört, dass die strategische Führungskraft eine klare Werteorientierung in der Organisation und bei ihren Mitgliedern etabliert und sie auch im Alltag vorlebt. Sie zeigt Verantwortungsgefühl für die gesamte Organisation sowie deren Aktivitäten. Die fünf Entwicklungsstufen stellen sich wie folgt dar: 
- Stufe 1: Der Basic Performer respektiert und akzeptiert die Werte und Führungsprinzipien der Organisation. Falls notwendig, übernimmt er Verantwortung. Darüber hinaus stellt er die Aufgabenerfüllung im Rahmen der Compliance-Regeln sicher.

- Stufe 2: Der Advanced Performer zeigt sich zusätzlich für ethisch-moralische Fragestellungen empfänglich. Er demonstriert Verantwortungsgefühl im Interesse der Organisation.

- Stufe 3: Der High Performer stellt sicher, dass die Werte der Organisation nicht nur bekannt sind, sondern sowohl in erfolgreichen als auch in schwierigen Zeiten durchgängig gelebt werden. Er fühlt sich für die Strategie der Organisation und deren Implikationen verantwortlich, explizit auch für über die Organisationsgrenzen hinausgehende externe Effekte. Er übernimmt zudem Verantwortung für die spezifischen Bedürfnisse aller Stakeholder und der Gesellschaft als Ganzes.

- Stufe 4: Der Outperformer gestaltet und prägt aktiv die Werte der Organisation. Er agiert als Vorbild für deren Mitglieder im Sinne des „ehrbaren Kaufmanns“. Er setzt sich nicht nur mit neuen gesellschaftlichen Ansprüchen auseinander, sondern managt sie auch positiv und aktiv. Er schafft eine ethisch orientierte Arbeitsumgebung und demonstriert in seiner Arbeit ein ausgeprägtes Augenmaß.

- Stufe 5: Der Over-the-top Performer übertreibt diese Verhaltensmuster, indem er die Verantwortung für alles und jeden regelrecht an sich reißt und dabei andere verdrängt. Verantwortung kann er nur schlecht abgeben. Er agiert dogmatisch und pedantisch und wird dadurch zum sprichwörtlichen Prinzipienreiter.

Mitarbeiter ermächtigen: Dies steht für die Kompetenz, als „Enabler“ zu fungieren und somit Kollegen und Mitarbeitern den Zugang zu ihrem Potenzial und ihrer bestmöglichen Leistung zu eröffnen. Ein Individuum mit dieser Kompetenz ist ein überzeugender und inspirierender Kommunikator, dem es gelingt, diverse Einheiten und Mitglieder der Organisation zu integrieren und Menschen gut anzuleiten. Die fünf Entwicklungsstufen stellen sich wie folgt dar:

- Stufe 1: Dem Basic Performer ist bewusst, dass auf allen Stufen gute Mitarbeiter benötigt werden. Er demonstriert die Fähigkeit, geeignete Mitarbeiter auszuwählen und für die Organisation zu verpflichten. Da er Mitarbeiter gut einschätzen kann, verfolgt er eine sinnvolle Besetzungs- und Beförderungspolitik.

- Stufe 2: Der Advanced Performer ist in der Lage, gute und starke Teams zusammenzustellen. Er wirkt auf eine integrative Art und Weise und fördert den Einsatz und die Arbeit in Teams. Er entwickelt das Personal und insbesondere die Talente der Organisation weiter, beispielsweise durch ausführliche Feedbacks oder direktes Coaching.

- Stufe 3: Der High Performer stellt zusätzlich sicher, dass die gesamte Organisation über ein tragfähiges System zur Personalentwicklung im Sinne der Strategie unterstützt wird. Er ist ein guter Kommunikator, der eine klare Botschaft auf überzeugende Art und Weise vermitteln kann. Er findet ein ausgewogenes Verhältnis zwischen Freiraum und Kontrolle in der Mitarbeiterführung. Darüber hinaus zeigt er die Fähigkeit und Bereitschaft zur Zusammenarbeit in unterschiedlichen Kontexten über die Grenzen zwischen diversen Organisationseinheiten und Teams hinweg.

- Stufe 4: Der Outperformer fördert grundsätzlich die übergreifende Zusammenarbeit in der Organisation. Darüber hinaus stärkt er seine Mitarbeiter, indem er Verantwortlichkeiten und Entscheidungsbefugnisse abgibt, um den Mitarbeitern die Gelegenheit zur 
bestmöglichen Leistung einzuräumen. Er überzeugt und inspiriert durch seine Persönlichkeit und ist dadurch auch in der Lage, die meisten Mitarbeiter für die Strategie und das entsprechende Engagement zu gewinnen.

- Stufe 5: Der Over-the-top Performer wiederum übertreibt diese positiven Aspekte. Da er zu viel an seine Mitarbeiter abgibt oder ihnen gar alles überlässt, handelt er nicht mehr als Vorbild. Indem er seinen Mitarbeitern immer bejahend und bestätigend gegenübersteht, geht er notwendigen Kontroversen aus dem Weg.

\section{(3) Sich selbst führen}

Die dritte Kompetenzkategorie „Sich selbst führen“ umfasst vier Teilkompetenzen, und zwar „Selbst-Reflexion“, „Entscheidungsstärke“， „Unternehmergeist“ sowie „Aufgeschlossenheit".

Selbst-Reflexion: Strategische Führungskräfte, die über die Kompetenz der Selbst-Reflexion verfügen, zeichnet aus, dass sie authentische, integre und in gewisser Weise bescheiden-zurückhaltende Personen sind, die sich und ihr Verhalten ständig reflektieren und hinterfragen. Dies tun sie mit der Intention, sich zu verbessern und weiterzuentwickeln. Die fünf Entwicklungsstufen stellen sich wie folgt dar:

- Stufe 1: Der Basic Performer akzeptiert Feedback und ist in der Lage, es anzunehmen. Er sieht die Notwendigkeit, an sich zu arbeiten und sich weiterzuentwickeln. Darüber hinaus kontrolliert er seine Emotionen und Gefühle.

- Stufe 2: Der Advanced Performer passt sich und sein Verhalten kontinuierlich an, um gute Leistungen zu erbringen. Er legt an sich selbst hohe Standards an, verfügt über eine angemessene Selbsteinschätzung. Seine Handlungen und Aktivitäten zeichnen sich durch ein hohes Maß an Integrität und Authentizität aus.

- Stufe 3: Der High Performer hinterfragt sich regelmäßig selbst und demonstriert die Bereitschaft, sich grundsätzlich zu verändern, zu lernen und sich weiterzuentwickeln. Er beobachtet aktiv die Reaktionen seiner Umwelt und anderer auf das eigene Verhalten, um dieses gegebenenfalls anzupassen. Er bemüht sich, in seinen Handlungen möglichst transparent zu sein.

- Stufe 4: Der Outperformer sucht das Feedback und lädt andere aktiv ein, ihn zu hinterfragen. Seine Handlungen sind von bescheidener Zurückhaltung geprägt. Dadurch erhält er eine Art öffentlicher Legitimation. Er zeigt ein feines Gespür für sich verändernde Anforderungen und Ausgangslagen und passt sich entsprechend an.

- Stufe 5: Der Over-the-top Performer übertreibt diese Verhaltensweisen, indem er extrem viel reflektiert und nachdenkt, was zu einem sehr zögerlichen Verhalten führt. Er hinterfragt sich und jede seiner Handlungen permanent und wird dadurch unfähig zu handeln. Er versucht ständig, jedem zu gefallen und es allen recht zu machen.

Entscheidungsstärke: Damit wird die Teilkompetenz bezeichnet, gute Entscheidungen unter Zeitdruck und Unsicherheit treffen zu können. Die Führungskraft ist konsistent in ihren Entscheidungen und konsequent in deren Umsetzung. Die fünf Entwicklungsstufen stellen sich wie folgt dar:

- Stufe 1: Der Basic Performer ist in der Lage, Entscheidungen unabhängig zu treffen. Er demonstriert Entschlusskraft. Auch bewahrt er bei einem herausfordernden Umfeld einen kühlen Kopf. 
- Stufe 2: Der Advanced Performer ist in der Lage, Entscheidungen auch unter Unsicherheit und Zeitdruck zu fällen. Er besitzt Vertrauen in seine Fähigkeit, die richtigen Entscheidungen zu treffen, und kann diese Entscheidungen anschließend auch umsetzen. Er demonstriert eine gewisse Konsistenz in seinen Entscheidungen, sowohl hinsichtlich der Strategie als auch in Bezug auf vorherige Entscheidungen.

- Stufe 3: Dem High Performer gelingt es zusätzlich, seine Entscheidungen flexibel anzupassen, wenn ein sich verändernder Kontext dies erfordert. Er bleibt bei Unsicherheit ruhig und steht zu seinen Entscheidungen. Wenn nötig, verfügt er über ein starkes Durchsetzungsvermögen. Er ist zudem in der Lage, aus falschen Entscheidungen zu lernen.

- Stufe 4: Der Outperformer zeichnet sich dadurch aus, dass sich die meisten seiner Entscheidungen rückblickend als korrekt und gut herausstellen. Nicht zuletzt deswegen wird er oft von anderen aufgesucht und um Rat und Lösungsvorschläge gebeten. Auch demonstriert er die Stärke, sich für Entscheidungen die nötige Zeit zu nehmen und, wenn notwendig, einen Schritt zurück zu machen.

- Stufe 5: Der Over-the-top Performer überschätzt seiner Entscheidungsfähigkeit. Er entscheidet zu schnell und ohne fundierte Informationen (soweit verfügbar). Er beharrt auf seinen Entscheidungen, drängt auf deren Umsetzung und übt dabei zu viel Druck auf seine Kollegen und Mitarbeiter aus.

Unternehmergeist: Eine strategische Führungskraft, die darüber verfügt, zeigt wahren unternehmerischen Geist, beispielsweise durch kreatives und innovatives Verhalten. Sie verfügt über strategische Weitsicht und ist bereit und in der Lage, Risiken kalkuliert einzugehen, um neue Geschäftsmöglichkeiten zu eröffnen und zu nutzen. Die fünf Entwicklungsstufen stellen sich wie folgt dar:

- Stufe 1: Der Basic Performer ist neuen Ideen und Erfahrungen gegenüber sehr offen. Er sieht häufig die positive Seite der Dinge. Dabei denkt und arbeitet er sehr problemlösungsorientiert.

- Stufe 2: Der Advanced Performer sucht aktiv nach Herausforderungen und nimmt sie auch an. Er energetisiert seine Umgebung und hat oft neue und einzigartige Ideen.

- Stufe 3: Der High Performer ist mutig und geht durchaus Risiken ein, wenn es neue Geschäftsfelder zu erschließen gilt. Er sieht Gelegenheiten und Chancen und nutzt sie konsequent. Er hat außerdem die Fähigkeit, neue Geschäftsfelder mit dem bestehenden Kontext zu verknüpfen.

- Stufe 4: Der Outperformer fördert Innovationen und Kreativität in der gesamten Organisation, beispielsweise indem er das Experimentieren ermöglicht und unterstützt. Er sucht aktiv nach neuen Lösungen und Verknüpfungen. Dabei denkt er strategisch, mit einem weiten Horizont und langfristig.

- Stufe 5: Der Over-the-top Performer hingegen übertreibt dieses Verhalten, indem er zu große Risiken eingeht. Er denkt nur noch an die Zukunft und neue Geschäftsfelder, vernachlässigt darüber aber das aktuelle Geschäft. Auf seine Mitarbeiter und Kollegen wirkt er dominant, aktivistisch und bedrängend.

Aufgeschlossenheit: Diese Teilkompetenz verweist auf eine weltoffene Führungskraft mit einem Blick für das große Ganze. Diese Führungskraft ist empathisch und kann aufmerksam zuhören. Die fünf Entwicklungsstufen stellen sich wie folgt dar: 
- Stufe 1: Der Basic Performer erkennt und reagiert auf die Gefühle und Sorgen seines Umfelds. Er ist in der Organisation sichtbar, da er für alle Mitglieder der Organisation und ihre Anliegen verfügbar ist. Er ist ein aktiver und geduldiger Zuhörer.

- Stufe 2: Der Advanced Performer verfügt über einen guten Zugang zu anderen Menschen und kann ihre Ideen, Meinungen und Gefühle nachvollziehen. Er demonstriert die Fähigkeit, in unterschiedlichen Kontexten mit unterschiedlichsten Menschen gut zusammenzuarbeiten. Zusätzlich ist er in der Lage, die eigene Organisation und das eigene Geschäft ganzheitlich zu betrachten.

- Stufe 3: Der High Performer akzeptiert die Möglichkeit von Fehlern und Fehlschlägen. Er interessiert sich aktiv für die Ideen, Meinungen und Gefühle der Personen seines Umfelds und fördert die Diversität in der Organisation. In dieser fungiert er als eine Art Membran, das heißt, er handelt empathisch und hört aktiv zu, sowohl den Mitgliedern der Organisation als auch den Stakeholdern.

- Stufe 4: Der Outperformer ist in der Lage, stärkere Verbindungen zu Mitgliedern der Organisation auf sämtlichen hierarchischen Ebenen herzustellen. Er versteht nicht nur sein Umfeld, sondern auch die tiefer liegenden Gründe für das Verhalten der ihn umgebenden Menschen. Er antizipiert zukünftige Herausforderungen mit Freude und sieht sie als Chancen.

- Stufe 5: Der Over-the-top Performer übertreibt diese Kompetenz, indem er zu empathisch ist und daher unfähig wird, Konflikte zu ertragen. Er hat keine eigene Meinung mehr und passt sich immer nur der Ansicht seines jeweiligen Gegenübers an. Er vertraut anderen Leuten zu sehr und vergisst sich selbst und seine eigene Position darüber. Er reibt sich zwischen unterschiedlichen Personen und deren abweichenden Meinungen auf.

Damit ist die Darstellung der neun Teilkompetenzen des MSF abgeschlossen. Im abschließenden Teil wollen wir uns der Anwendung des Modells zuwenden.

\section{Anleitung zur Durchführung von Bewertungen}

In diesem Abschnitt soll eine Möglichkeit aufgezeigt werden, wie das MSF in einer konkreten Evaluationssituation angewendet werden kann. Dabei sollte das Vorgehen in jedem Fall der konkreten Situation angepasst werden. Unveränderlich sind jedoch die drei Kompetenzkategorien mit den neun Teilkompetenzen.

Es empfiehlt sich unserer Erfahrung nach, ein Assessment in vier Prozessschritte zu untergliedern. Exemplarisch soll dies im Folgenden am Beispiel einer zu besetzenden Führungsposition geschehen:

1. Vorbereitung der Interviews

2. Erstellung eines Zielprofils und Kandidatenansprache

3. Durchführung der Interviews

4. Beurteilung der Kandidaten und Abgabe einer Empfehlung

\subsection{Vorbereitung der Interviews}

Im ersten Schritt geht es darum, dass der Interviewer sich mit dem Modell vertraut macht, sodass er es auch kompetent anwenden kann. Es ist sicherzustellen, dass er die drei Kompetenzkategorien mit den neun Teilkompetenzen kennt und weiß, was sie bedeuten. Auch 
sollte er die Verhaltensindikatoren jeder Teilkompetenz gut kennen und verstehen, um ihre jeweilige Ausprägung im Assessmentprozess benennen zu können.

Unterstützend werden ihm folgende Dokumente an die Hand gegeben werden: (a) eine detaillierte Beschreibung des Interviewprozesses auf der Basis des MSF, (b) eine detaillierte Beschreibung des MSF zusammen mit einer Kurzfassung (One Pager) sowie (c) ein Template für den Assessment-Report, der - wie in Abbildung 4 dargestellt - eine Zusammenfassung der Assessments enthält sowie eine Analyse jeder einzelnen der neun Teilkompetenzen. Das Template in Abbildung 4 wird sowohl für Schritt 2 als auch für Schritt 4 herangezogen, sodass ein systematischer Vergleich zwischen dem Zielprofil und dem Profil des Kandidaten möglich wird. 
Bekemeier/Meynhardt/Müller-Stewens | Was von Entscheidungsträgern erwartet wird

\begin{tabular}{|c|c|c|c|c|c|c|c|c|c|c|c|c|c|}
\hline \multicolumn{14}{|c|}{ Strategische Führungskompetenz } \\
\hline & \multicolumn{3}{|c|}{$\begin{array}{l}\text { Basic } \\
\text { Performer }\end{array}$} & \multicolumn{3}{|c|}{$\begin{array}{l}\text { Advanced } \\
\text { Performer }\end{array}$} & \multicolumn{3}{|c|}{$\begin{array}{l}\text { High } \\
\text { Performer }\end{array}$} & \multicolumn{3}{|c|}{$\begin{array}{c}\text { Out- } \\
\text { performer }\end{array}$} & $\begin{array}{r}\text { Over-the-top } \\
\text { Performer }\end{array}$ \\
\hline \multicolumn{14}{|c|}{ Die Organisation führen } \\
\hline \multirow{2}{*}{$\begin{array}{l}\text { Strategische } \\
\text { Orientierung geben }\end{array}$} & \multicolumn{3}{|c|}{ Stufe 1} & \multicolumn{3}{|c|}{ Stufe 2} & \multicolumn{3}{|c|}{ Stufe 3} & \multicolumn{3}{|c|}{ Stufe 4} & Stufe 5 \\
\hline & 0 & 1 & + & - & 2 & + & - & 3 & + & - & 4 & + & 5 \\
\hline \multirow{2}{*}{$\begin{array}{l}\text { Die Organisation } \\
\text { anpassen }\end{array}$} & \multicolumn{3}{|c|}{ Stufe 1} & \multicolumn{3}{|c|}{ Stufe 2} & \multicolumn{3}{|c|}{ Stufe 3} & \multicolumn{3}{|c|}{ Stufe 4} & Stufe 5 \\
\hline & 0 & 1 & + & - & 2 & + & - & 3 & + & - & 4 & + & 5 \\
\hline \multirow{2}{*}{$\begin{array}{l}\text { Spannungen } \\
\text { managen }\end{array}$} & \multicolumn{3}{|c|}{ Stufe 1} & \multicolumn{3}{|c|}{ Stufe 2} & \multicolumn{3}{|c|}{ Stufe 3} & \multicolumn{3}{|c|}{ Stufe 4} & Stufe 5 \\
\hline & 0 & 1 & + & - & 2 & + & - & 3 & + & - & 4 & + & 5 \\
\hline
\end{tabular}

\begin{tabular}{|c|c|c|c|c|c|c|c|c|c|c|c|c|c|}
\hline \multicolumn{14}{|c|}{ Mitarbeiter führen } \\
\hline \multirow{2}{*}{$\begin{array}{l}\text { Verantwortung } \\
\text { übernehmen }\end{array}$} & \multicolumn{3}{|c|}{ Stufe 1} & \multicolumn{3}{|c|}{ Stufe 2} & \multicolumn{3}{|c|}{ Stufe 3} & \multicolumn{3}{|c|}{ Stufe 4} & Stufe 5 \\
\hline & 0 & 1 & + & - & 2 & + & - & 3 & + & - & 4 & + & 5 \\
\hline \multirow{2}{*}{$\begin{array}{l}\text { Mitarbeiter } \\
\text { ermächtigen }\end{array}$} & \multicolumn{3}{|c|}{ Stufe 1} & \multicolumn{3}{|c|}{ Stufe 2} & \multicolumn{3}{|c|}{ Stufe 3} & \multicolumn{3}{|c|}{ Stufe 4} & Stufe 5 \\
\hline & 0 & 1 & + & - & 2 & + & - & 3 & + & - & 4 & + & 5 \\
\hline
\end{tabular}

\section{Sich selbst führen}

\begin{tabular}{|c|c|c|c|c|c|c|c|c|c|c|c|c|c|}
\hline \multirow{2}{*}{$\begin{array}{l}\text { Selbstreflektiv } \\
\text { handeln }\end{array}$} & \multicolumn{3}{|c|}{ Stufe 1} & \multicolumn{3}{|c|}{ Stufe 2} & \multicolumn{3}{|c|}{ Stufe 3} & \multicolumn{3}{|c|}{ Stufe 4} & Stufe 5 \\
\hline & 0 & 1 & + & - & 2 & + & - & 3 & + & - & 4 & + & 5 \\
\hline \multirow{2}{*}{$\begin{array}{l}\text { Entscheidungs } \\
\text { stärke zeigen }\end{array}$} & \multicolumn{3}{|c|}{ Stufe 1} & \multicolumn{3}{|c|}{ Stufe 2} & \multicolumn{3}{|c|}{ Stufe 3} & \multicolumn{3}{|c|}{ Stufe 4} & Stufe 5 \\
\hline & 0 & 1 & + & - & 2 & + & - & 3 & + & - & 4 & + & 5 \\
\hline \multirow{2}{*}{$\begin{array}{l}\text { Unternehmergeist } \\
\text { leben }\end{array}$} & \multicolumn{3}{|c|}{ Stufe 1} & \multicolumn{3}{|c|}{ Stufe 2} & \multicolumn{3}{|c|}{ Stufe 3} & \multicolumn{3}{|c|}{ Stufe 4} & Stufe 5 \\
\hline & 0 & 1 & + & - & 2 & + & - & 3 & + & - & 4 & + & 5 \\
\hline \multirow{2}{*}{$\begin{array}{l}\text { Aufgeschlossen } \\
\text { sein }\end{array}$} & \multicolumn{3}{|c|}{ Stufe 1} & \multicolumn{3}{|c|}{ Stufe 2} & \multicolumn{3}{|c|}{ Stufe 3} & \multicolumn{3}{|c|}{ Stufe 4} & Stufe 5 \\
\hline & 0 & 1 & + & - & 2 & + & - & 3 & + & - & 4 & + & 5 \\
\hline
\end{tabular}

Abb. 4: Template für die Zusammenfassung des Zielprofils beziehungsweise des Assessments

\subsection{Erstellung eines Zielprofils und Kandidatenansprache}

Im zweiten Schritt wird ein Zielprofil der Wunsch-Führungskraft erstellt, die für die zu besetzende Stelle gesucht wird. Dies geschieht zusammen mit den Personen, die für diese Stelle zuständig sind und über ihre Besetzung entscheiden. 
Herausforderung ist es hier, die Unternehmensstrategie in Form von Anforderungen an die Kandidaten in die neun Teilkompetenzen zu „übersetzen“: Was muss diese Person können, um die Strategie vorzuleben und umzusetzen oder sogar eine geeignete Strategie erst noch zu entwickeln? Was ist der spezifische Kontext der Stelle, beispielsweise das unternehmerische Umfeld oder die Zusammensetzung des Führungsteams? Dazu kann das Template für den Assessment-Report aus Abbildung 4 herangezogen werden, das es auszufüllen gilt. Zur Festlegung der Zielausprägungen der einzelnen Teilkompetenzen ist auch eine Kontextualisierung der Verhaltensindikatoren erforderlich. Falls unbedingt erforderlich, können die neun Teilkompetenzen um fallspezifische Anforderungen erweitert werden.

Auf der Basis des Zielprofils kann nun sowohl intern als auch extern nach geeigneten Kandidaten Ausschau gehalten werden. Die, die identifiziert wurden und an einem Gespräch interessiert sind, werden - zum Beispiel mittels einer Videokonferenz - einer kurzen Vorauswahl unterzogen, um sicherzustellen, dass sie ins Suchspektrum fallen. Danach erfolgt die Einladung zum persönlichen Interview.

\subsection{Durchführung der Interviews}

Das Interview ist in drei Phasen untergliedert: Vorbereitung, Durchführung und Nachbereitung. Vor dem Interview hat sich der Interviewer intensiv mit den durch den Kandidaten eingereichten Unterlagen auseinanderzusetzen, um sich vor dem Hintergrund der neun geforderten Teilkompetenzen ein erstes Bild von ihm zu machen.

Ziel des Interviews ist es dann, möglichst reichhaltige Beschreibungen und Reflexionen $\mathrm{zu}$ aus Sicht des Kandidaten strategisch bedeutsamen Situationen und Vorkommnissen zu erhalten. Dazu wählt er drei solcher Ereignisse aus: Eines davon hat er aus seiner Sicht erfolgreich gemeistert, eines nicht erfolgreich und ein drittes hatte besonders starke Auswirkungen auf die Organisation, in der er dabei tätig war. Eine Alternative bestünde darin, dass das dritte Ereignis durch den Interviewer auf der Basis der eingereichten Unterlagen vorgegeben wird. Die ausgewählten Entscheidungssituationen sollten nicht zu komplex sein, da ihre Rekonstruktion im Gespräch zu zeitaufwendig werden würde.

Nun geht es darum, dass der Interviewer den Interviewten ermuntert, jedes Ereignis ausführlich zu beschreiben und zu kommentieren: Was macht es überhaupt strategisch bedeutsam? Worin bestand die genaue Herausforderung? Was hatte dazu geführt? Welche Herangehensweise wurde gewählt? Wie hat sich der Prozess entfaltet? Mit welchen Überraschungen war man dabei konfrontiert? Gab es Konflikte und wie ist man mit ihnen umgegangen? Wie wurde entschieden? Was machte die Entscheidung schwierig? Was waren die Unwägbarkeiten bei der Entscheidung? Was waren Gedanken und Gefühle in der Situation? Was waren Output und Outcome der Entscheidung? Wie beurteilt man das eigene Verhalten im Nachhinein? Usw. Das gleiche Vorgehen wird dann bei den anderen beiden Ereignissen praktiziert.

Im Hinterkopf muss der Interviewer behalten, dass er Informationen bekommt, um am Ende Aussagen möglichst zu allen neun Teilkompetenzen treffen zu können. Das geschieht am einfachsten, wenn es gelingt, nach und nach für den Kandidaten typische Verhaltensmuster ausfindig zu machen.

Derartige Interviews sind als gemeinsamer Entdeckungsprozess zu verstehen. Bei den Erzählungen heißt es für den Interviewer, das Gespräch mit gezielten Fragen zu steuern, sich aber ansonsten eher im Hintergrund zu halten und aufmerksam zuhören. Auch muss der Interviewer sich der Tatsache bewusst sein, dass Fragen nach Erfolg und Misserfolg emo- 
Bekemeier/Meynhardt/Müller-Stewens | Was von Entscheidungsträgern erwartet wird

tional sehr aufwühlend sein können. Sein Ziel ist es, in Erfahrung zu bringen, wie der Kandidat seine Führungsaufgabe erledigt hat: Was und wie dachte und fühlte er in der jeweiligen Situation? Wie hat er über andere beteiligte Personen gedacht und geurteilt? Was hat in der Situation sein Handeln und Entscheiden motiviert?

Bei der Steuerung des Interviews ist darauf zu achten, dass der Fokus nicht auf den Fakten liegt, sondern auf den dazugehörigen Reflexionen. Wichtig ist auch, dass die zeitliche Abfolge sauber erfasst wird. Vielleicht lassen sich ja auch schon bestimmte Verhaltensmuster entdecken. Unbedingt zu vermeiden ist, dass direkt nach Kompetenzen gefragt wird. Auch sollte nicht direkt in den Kategorien des Modells argumentiert oder gefragt werden. Es ist konkret nachzuhaken, wenn zu abstrakt erzählt wird.

\subsection{Beurteilung der Kandidaten und Abgabe einer Empfehlung}

Nach dem Interview muss die Beurteilung des Kandidaten vorgenommen werden. Auf der Basis der im Interview getroffenen Aussagen müssen nun die Ausprägungen auf der Kompetenzskala aller neun Teilkompetenzen festgelegt werden, wobei es hilfreich ist, wenn das Interview mit Zustimmung des Teilnehmers aufgezeichnet werden konnte.

Danach wird die Beurteilung des Kandidaten aus dem Interview mit dem Zielprofil der zu besetzenden Stelle verglichen: Wo besteht eine hohe Übereinstimmung? Wo gibt es erhebliche Differenzen? Auf dieser Basis können auch alle Kandidaten untereinander hinsichtlich ihrer Eignung miteinander verglichen werden, was die Grundlage für die Abgabe einer wohlbegründeten Empfehlung an den Auftraggeber ist.

Besetzt einer der interviewten Kandidaten die ausgeschriebene Stelle, so kann das Ergebnis des Profilvergleichs als Grundlage für ein erstes Entwicklungsgespräch herangezogen werden.

\section{Schlussbetrachtung: Ein Leitkonzept, aber kein Dogma}

Mit der Nutzung des MSF werden Unternehmen in die Lage versetzt, ihre Strategie mit der Führung auf der Ebene des einzelnen Akteurs zu verknüpfen. So soll der Geschäftskontext mit den spezifischen Anforderungen an eine Position unter Bezug auf das Zielprofil und die Fähigkeiten eines potenziellen Kandidaten weitmöglichst zur Deckung gebracht werden. Mit diesem Modell können Strategen in ihrer Kompetenz in einer standardisierten Form beurteilt und untereinander vergleichen werden. Auch können auf der Basis einer Auditierung gezielt Förder- und Unterstützungsmaßnahmen ergriffen werden.

Mit dem hier entwickelten Modell ist allerdings keineswegs der Anspruch verbunden, beruflichen Erfolg anhand der wenigen Faktoren des Modells abschließend erklären zu wollen. Mit diesen Kompetenzen allein lässt sich selbstverständlich nicht alles erklären. Die Realität ist normalerweise um einiges komplexer und die Arbeit der Strategen ist meist keine simple Angelegenheit; sie ist kompliziert und vielschichtig und verlangt, dass wir dabei tief und fest in der Realität verankert sind. Das Modell möchte eine Orientierungshilfe für Manager und Führungskräfte sein, ohne den zuvor angesprochenen Anspruch einer vollständigen Erklärung erfolgreicher Performanz zu liefern. Vielmehr sollen Strategen damit ihren Platz in der sie umgebenden organisatorischen Gemeinschaft und der Gesellschaft als Ganzes suchen. Dort haben sie ihre moralischen Pflichten zu erfüllen und für das 
Ganze ein Zugewinn zu sein. ${ }^{34}$ Die Energie für unser aller Vorankommen beziehen wir aus unseren zwischenmenschlichen Interaktionen mit unseren Anspruchsgruppen - nicht aus einer isolierten Optimierung unserer selbst. ${ }^{35}$

Das Forschungsdesign selber impliziert natürlich gewisse Einschränkungen in der Gültigkeit. So führt die Untersuchung dispositionaler Strukturen immer zu Schwierigkeiten, da sich selbige nicht direkt beobachten lassen und wir nur Rückschlüsse aus demonstriertem Verhalten ziehen können. Darüber hinaus ist auch die Performanz individueller Führungskräfte sowie deren Beurteilung mit diversen Herausforderungen verbunden wie zuvor dargelegt. Auch wird es in vielen Fällen notwendig sein, das Modell an die unterschiedlichsten Kontexte anzupassen. Wie zuvor dargelegt gibt es eine grosse Anzahl relevanter kontextualer Faktoren wie beispielsweise die Berufsgruppe, die Branche, die jeweilige Organisation oder auch die Verknüpfungen zu den jeweiligen Führungsteams.

Trotz dieser Einschränkungen meinen wir, dass es sich lohnt, mit einem möglichst konkreten Verständnis einer strategischen Führungskompetenz, wie sie das MSF bietet, als eine Art Leitidee zu arbeiten. Dabei sollte man auch offen sein, sich bei guten Gründen vom Modell wegzubewegen. So sehen wir in dem an den Kontext anzupassenden Modell eine pragmatische Hilfestellung und ein nützliches Instrument, das uns einen systematischen Zugang zur Thematik gibt.

\section{Danksagung}

Dieser Beitrag beruht auf den Erkenntnissen aus einem mehrjährigen Forschungsprojekt der Universität St. Gallen und der internationalen Executive-Search-Firma Boyden. Aufseiten der Universität haben mitgearbeitet die Autoren dieses Beitrags; aufseiten Boydens waren dies insbesondere Dr. Dirk Friederich, Trina Gordon, Gray Hollet und Armin Meier sowie die Boyden-Partner, die uns für die Durchführung der Interviews zur Verfügung standen. Ihnen allen gilt unser aufrichtiger Dank. Ohne ihre Beiträge wäre dieses Projekt in dieser Form nicht möglich gewesen. Danken wollen wir auch den Reviewern dieses Beitrags für deren konstruktive Verbesserungsvorschläge.

\section{Literatur}

Alldredge, M. E./Nilan, K. J. (2000): 3M's leadership competency model: An internally developed solution, in: Human Resource Management, 39(2,3), 133-145.

Athey, T. R./Orth, M. S. (1999): Emerging competency methods for the future, in: Human Resource Management, 38(3), 215-226.

Bekemeier, S. (2016): Strategic Leadership Competence - Konzeptualisierung und Erhebung einer Kernkompetenz von Führungskräften, Dissertation Universität St. Gallen.

Borman, W. C./Brush, D. H. (1993): More progress toward a taxonomy of managerial performance requirements, in: Human Performance, 6(1), 1-21.

Boyatzis, R. E. (1982): The competent manager: A model for effective performance. New York: Wiley.

34 Vgl. zur Verbindung der Motivation des einzelnen Managers zum größeren Ganzen Meynhardt (2018) und Müller-Stewens (2019), Kapitel 1.

35 Vgl. dazu den Beitrag „Der Ratgeber, der keiner sein will“ von Rudolf Herrmann über den dänischen Philosophen Svend Brinkmann in der NZZ vom 16.06.2018. 
Boyatzis, R. E. (2009): Competencies as a behavioral approach to emotional intelligence, in: Journal of Management Development, 28(9), 749-770.

Catano, V. M./Darr, W./Campbell, C. A. (2007): Performance appraisal of behavior-based competencies: A reliable and valid procedure, in: Personnel Psychology, 60, 201-230.

Charmaz, K. (2006): Constructing grounded theory: A practical guide through qualitative analysis, Los Angeles: SAGE.

Dreyfus, S. E. (2004): The five-stage model of adult skill acquisition: in: Bulletin of Science, Technology and Society, 24(3), 177-181.

Erpenbeck, J. (2012): Zwischen exakter Nullaussage und vieldeutiger Beliebigkeit: Hybride Kompetenzerfassung als künftiger Königsweg, in: Erpenbeck, J. (Hrsg.): Kompetenzmanagement in der Praxis, Bd. 6, Der Königsweg zur Kompetenz. Grundlagen qualitativ-quantitativer Kompetenzerfassung, Münster: Waxmann, 7-42.

Erpenbeck, J./von Rosenstiel, L. (Hrsg., 2007): Handbuch Kompetenzmessung: Erkennen, verstehen und bewerten von Kompetenzen in der betrieblichen, pädagogischen und psychologischen Praxis, 2. Auflage, Stuttgart: Schäffer-Poeschel.

Erpenbeck, J./von Rosenstiel, L./Grote, S. (Hrsg., 2013): Kompetenzmodelle von Unternehmen: Mit praktischen Hinweisen für ein erfolgreiches Management von Kompetenzen, Stuttgart: SchäfferPoeschel.

Finkelstein, S./Hambrick, D. C./Cannella, A. A. (2009): Strategic leadership; Theory and research on executives, top management teams and boards, Oxford: Oxford University Press.

Flanagan, J. C. (1954): The critical incident technique, in: Psychological Bulletin, 51(4), 327-359.

Gebert, D./Boerner, S./Lanwehr, R. (2003): The risks of autonomy: Empirical evidence for the necessity of a balance management in promoting organizational innovativeness, in: Creativity \& Innovation Management, 12(1), 41-49.

Glaser, B. G./Strauss, A. L. (1999): The discovery of grounded theory: Strategies for qualitative research, New Brunswick, NJ: Aldine Transaction.

Habermas, J. (1995): Theorie des kommunikativen Handelns, Frankfurt a. M.: Suhrkamp.

Hamel, G. (1994): The Concept of Core Competence, in: Hamel, G./Heene, A. (Hrsg.): The Strategic management series. Competence-based competition, Chichester, NY: Wiley, 11-34.

Hoffman, B. J./Woehr, D. J./Maldagen-Youngjohn, R./Lyons, B. D. (2011): Great man or great myth? A quantitative review of the relationship between individual differences and leader effectiveness, in: Journal of Occupational and Organizational Psychology, 84(2), 347-381.

International Group of Controlling (Hrsg., 2015): Controller-Kompetenzmodell. Ein Leitfaden für die moderne Controller-Entwicklung mit Muster-Kompetenzprofilen, Freiburg: Haufe-Lexware GmbH \& Co. KG.

Ireland, R. D./Hitt, M. A. (2005): Achieving and maintaining strategic competitiveness in the 21st century: The role of strategic leadership, in: Academy of Management Executive, 19(4), 63-77.

Johnson, G./Melin, L./Whittington, R. (2003): Micro strategy and strategizing: Towards an activitybased view, in: Journal of Management Studies, 40(1), 3-22.

Kolb, D. A. (1984): Experiential learning: Experience as the source of learning and development, Englewood Cliffs, NJ: Prentice Hall.

Krumm, S./Mertin, I./Dries, C. (2012): Kompetenzmodelle, Göttingen et al.: Hogrefe.

Kurz, R./Bartram, D. (2002): Competency and individual performance: Modelling the world of work, in: Robertson, I. T./Callinan, M./Bartram, D. (Hrsg.): Organizational effectiveness. The role of psychology, Chichester, NY: Wiley, 227-255.

Die Unternehmung, 74. Jg., 1/2020 


\section{Beiträge}

Levenson, A. R./Van der Stede, W. A./Cohen, S. G. (2006): Measuring the relationship between managerial competencies and performance, in: Journal of Management, 32(3), 360-380.

McClelland, D. C. (1973): Testing for competence rather than for „intelligence“, in: American Psychologist, 28(1), 1-14.

Meynhardt, T. (2004): Wertwissen: Was Organisationen wirklich bewegt, Münster: Waxmann.

Meynhardt, T. (2007): Zur Verbindung zwischen unternehmerischer Kernkompetenz und individueller Kompetenz: Zusammen denken, getrennt analysieren, gemeinsam entwickeln, in: Barthel, E./ Erpenbeck, J./Hasebrook, J./Zawacki-Richter, O. (Hrsg.): Kompetenzkapital heute. Wege zum integrierten Kompetenzmanagement, Frankfurt a. M.: Frankfurt School Verlag, 293-325.

Meynhardt, T. (2018): Public value: Value creation in the eyes of society, in: Lindgreen, A./KoenigLewis, N./Kitchener, M./Brewer, J./Moore, M./Meynhardt, T. (Hrsg.): Managing public value, London: Routledge.

Molleman, E./van der Vegt, G. S. (2007): The performance evaluation of novices: The importance of competence in specific work activity clusters, in: Journal of Occupational and Organizational Psychology, 80(3), 459-478.

Müller-Stewens, G. (2019): Die neuen Strategen. Gestalter der Unternehmenszukunft, Stuttgart: Schaeffer-Poeschel Verlag.

Penrose, E. (1959): The theory of the growth of the firm, Oxford.

Popper, K. R. (2004): Alle Menschen sind Philosophen. München/Zürich: Piper.

Prahalad, C. K./Hamel, G. (1990): The core competence of the corporation, in: Harvard Business Review, 68(3), 79-91.

Ramo, L. G./Saris, W. E./Boyatzis, R. E. (2009): The impact of social and emotional competencies on effectiveness of Spanish executives, in: Journal of Management Development, 28(9), 771793.

Rarrek, A./Werner, E. P. (2012): Die Krux mit den Fähigkeiten, in: Erpenbeck, J. (Hrsg.): Der Königsweg zur Kompetenz. Grundlagen qualitativ-quantitativer Kompetenzerfassung, Münster: Waxmann, 43-52.v

Reed, K. K./Lubatkin, M./Srinivasan, N. (2006): Proposing and testing an intellectual capital-based view of the firm, in: Journal of Management Studies, 43(4), 867-893.

Rothaermel, F. T./Hess, A. M. (2007): Building dynamic capabilities: Innovation driven by individual-, firm-, and network-level effects, in: Organization Science, 18(6), 898-921.

Russell, C. J. (2001): A longitudinal study of top-level executive performance, in: Journal of Applied Psychology, 86(4), 560-573.

Sanchez, J. I./Levine, E. L. (2009): What is (or should be) the difference between competency modeling and traditional job analysis?, in: Human Resource Management Review, 19(2), 53-63.

Scherm, M. (2014): Kompetenzfeedbacks: Selbst- und Fremdbeurteilung beruflichen Verhaltens, Göttingen et al.: Hogrefe.

Sonntag, K. (2006): Ermittlung tätigkeitsbezogener Merkmale: Qualifikationsanforderungen und Voraussetzungen menschlicher Aufgabenbewältigung, in Sonntag, K. (Hrsg.): Personalentwicklung in Organisationen, 3. Auflage, Göttingen et al.: Hogrefe, 206-234.

Sonntag, K./Stegmaier, R. (2006): Personalentwicklung und Unternehmensperformance - Eine Evaluationsperspektive für das Human Resource Management, in: Sonntag, K. (Hrsg.): Personalentwicklung in Organisationen, 3. Auflage, Göttingen et al.: Hogrefe, 389-410.

Spencer, L. M./Spencer, S. M. (1993): Competence at work: Models for superior performance. New York: Wiley. 
Bekemeier/Meynhardt/Müller-Stewens | Was von Entscheidungsträgern erwartet wird

Strauss, A. L./Corbin, J. M. (1998): Basics of qualitative research: Techniques and procedures for developing grounded theory, 2. Auflage, Thousand Oaks: Sage Publications.

Sudsakorn, T./Swierczek, F. W. (2009): Management competencies: a comparative study between Thailand and Hong Kong, in: Journal of Management Development, 28(7), 569-580.

Teece, D. J. (2014): The foundations of enterprise performance: Dynamic and ordinary capabilities in an (economic) theory of firms, in: Academy of Management Perspectives, 28(4), 328-352.

Teece, D. J./Pisano, G./Shuen, A. (1997): Dynamic capabilities and strategic management, in: Strategic Management Journal, 18(7), 509-533.

Tett, R. P./Burnett, D. D. (2003): A personality trait-based interactionist model of job performance, in: Journal of Applied Psychology, 88(3), 500-517.

Thomas, A. S./Ramaswamy, K. (1996): Matching managers to strategy: Further tests of the Miles and Snow typology, in: British Journal of Management, 7(3), 247-261.

Whittington, R. (2001): What is strategy - and does it matter?, 2. Auflage, London: Thomson Learning.

Wunderer, R./Bruch, H. (2000): Umsetzungskompetenz: Diagnose und Förderung in Theorie und Unternehmenspraxis, München: Vahlen.

Zaccaro, S. J. (2007): Trait-Based Perspectives of Leadership, in: American Psychologist, 62(1), 616.

Zwell, M. (2000): Creating a culture of competence, New York: Wiley.

Sebastian Bekemeier, Dr., ist Consultant \& Learning Partner bei der ABB Schweiz AG

Anschrift: Glatttalstrasse 110, CH-8052 Zürich (Schweiz)

Timo Meynhardt, Prof. Dr., ist Inhaber des Dr.-Arend-Oetker-Lehrstuhls für Wirtschaftspsychologie und Führung an der Leipzig Graduate School of Management und Managing Director des Center for Leadership and Values in Society an der Universität St. Gallen.

Anschrift: HHL Leipzig Graduate School of Management, Jahnallee 59, D-04109 Leipzig (Deutschland)

Günter Müller-Stewens, Prof. em. Dr., ist emeritierter Professor und Direktor am Institut für Betriebswirtschaft der Universität St. Gallen

Anschrift: Institut für Betriebswirtschaft,Universität St.Gallen (IfB-HSG), Dufourstrasse 40a, CH-9000 St.Gallen (Schweiz). 